\title{
Ueber die Einwirkung verdünnter Säuren und Salzlösungen auf Gelatine.
}

\author{
Von Henry R. Procter.
}

(Eingegangen am 29. Okt. th. 10. Dez. 1910)

Die Untersuchung, die den Gegenstand dieser Abhandlung bildet, wurde 1897 begonnen und ein großer Teil der Experimente wurde 1898 gemeinsam mit Herrn Richard Poget ausgeführt, der, wie wir hofften, an der Herausgabe der Arbeit teilnehmen sollte Verschiedene Umstände verhinderten jedoch die gemeinsame Vollendung und so wurde die Arbeit unter verschiedener Assistenz mit Unterbrechungen bis heute weitergeführt; obwohl noch manche Fragen ungelöst bleiben müssen, scheint es im Hinblick auf das gegenwärtig herrschende Interesse für Fragen der Kolloidchemie wünschenswert, mit der Veröffentlichung des bis jetzt Vollendeten nicht länger zu zögern.

Die Untersuchung wurde in der Hoffnung unternommen, daß das Studium verhältnismäßig einfacher Fälle kolloider Quellung und Entquellung einiges Licht auf das komplizierte Phänomen des Gerbprozesses werfen würde; besonders aber sollten die sehr merkwürdigen Resultate der Behandlung der Häute mit Säuren und Salzen, die als „Pickeln" bekannt ist, und die mineralischen Gerbprozesse, bei denen man Säuren und Salze verwendet, aufgeklärt werden; aber bei weiterem Fortschreiten unserer Versuche zeigte es sich, dab viel wichtige wissenschaftliche Gebiete berührt werden muRten, die in ihrer Geșamtheit die ganze Theorie der Quellung der Kolloide einschlieBen.

Der ProzeB des Pickelns besteht im wesentlichen in der Behandlung der Haut mit einem schr verdünnten Bad von Schwefelsäure, in dem die Bindegewebsfasern stark anschwellen, und im darauffolgenden Eintauchen in eine konzentrierte Neutralsalzlösung, in der dann nicht nur die Schwellung verschwindet, sondern die Fasern auch stark entwässert werden, so daß sich die Halıt in eine Art Leder verwandelt. Da ohne vorhergehende Säurebehandlung sogar gesättigte Kochsalzlösungen keine dehydratisierende Wirkung haben, erscheint der obige Befund auf den ersten Blick unverständlich.

Der Quellungsprozik wird bei der Haut durch ihre anatumisrhe Siruklur kompliziert, dic es gestattet, Flïssigkeiten nicht nur durch 
kolloide Quellung, sondern auch kapillar in den Zwischenräumen zwischen den Fasern aufzunehmen, und es war klar, daB quantitative Untersuchungen erst dann gemacht werden konnten, wenn Mittel gefunden waren, diese beiden Vorgänge $z u$ trenten. Glücklicherweise verhält sich Gelatine in bezug auf die Quellung zumindest qualitativ identisch mit Hautfasern, und die nahe chemische Verwandtschaft der beiden Stoffe rechtfertigt die Annahme, daB dabei die gleichen chemischen Affinitäten wirken, während durch die Abwesenheit einer Struktur kapillare Adsorption ausgeschlossen ist. Vergleichende Experimente bestätigten diese angenommene Gleichheit des Verhaltens; da die diesbezüglichen Versuche nur als vorbereitende Untersuchungen gedacht waren, wurde die gewöhnliche, im Handel erhältliche, dünnplattige Gelatine verwendet. Aus demselben Grunde und um eine Komplizierung der Arbeit zu vermeiden, wurden geringe Aenderungen der Laboratoriumstemperatur und andere sekundăre Störungen, wie anhaftende Feuchtigkeit, nicht berücksichtigt und es wurde eine Arbeitsmethode gewählt, die ein verhältnismäßig schnelles Arbeiten ermöglichte: Platten von dünner französischer Gelatine der reinsten Sorte wurden in lufttrockenem Zustande in Teile von etwa $1 \mathrm{~g}$ Gewicht geschnitten, in die betreffenden Lösungen gelegt und, nachdem sie soweit wie möglich von anhaftender Feuchtigkeit befreit worden waren, die Gewichtszunahme bestimmt; sodann wurde sowohl die Gelatine als die rückständigen Lösungen auf ihren Säure- und Salzgehalt analysiert und das Ganze auf aschenfreie, bei $110^{\circ}$ getrocknete Gelatine und auf Milligramm-Moleküle Elektrolyte pro.Gramm Gelatine bezogen. Die lufttrockene Gelatine enthielt bei den früheren Versuchen 16,07 Proz. Feuchtigkeit, die praktisch konstant blieb, da die Gelatine in verstopften Flaschen aufbewahrt wurde, und 1,19 Proz. Asche, die hauptsächlich aus Kalk und Spuren von Sulfiden und Phosphaten bestand. Es war also wohl eine Knochengelatine. Für alle früheren Versuche war dieselbe Gelatine verwendet worden, doch für einige der späteren Versuche gelangten andere Gelatinen derselben Art aber nicht von genau der gleichen Zusammensetzung zur Verwendung. Dies mag einige Variationen zwischen verschiedenen Versuchsserien erklären, während die einzelnen Versuchsreihen in der Regel sehr übereinstimmende Kurven ergeben

\section{Die Quellung von Gelatine in Wasser.}

Wieweit eine Gelatine in kaltem Wasser bei einer gegebenen Temperatur quellen kann, ist zum großen Teil eire spezifische Eigen- 
schaft der einzelnen Sorten, die beeinfluBt wird durch den Gehalt an Produkten teilweise hydrolysierter Gelatine, wie sie in der kafuflichen Gelatine immer vorhanden sind. Ihre Bildung labt sich tatsichlich nicht vollkommen vermeiden, da sie immer in gewisser Menge entstehen, wenn die Gelatinegallerte bis zum Schmelzen erhitzt wird, und sie sind unzweifelhaft die Hauptursache der Variationen in Charakter der Gelatinereaktionen, die der sogenatunten "Vorgeschichte" der Gelatine zugeschrieben werden. Spuren löslicher Elektrolyte wirken übrigens gleichfalls, und zwar osmotisch, vielleicht aber auch chemisch auf sie ein. Daß Gelatine und andere gelatinose Substanzen nicht bis zur Unendlichkeit quellen, sondern bloB kolloide Lösungen bilden, wie Gummi und Dextrin, ist auf die feste, aber elastische Struktur zurückzufiahren, die sich beim Erstarren bildet und deren Kohäsion schlieblich der Adhăsion zwischen Gelatine und Wasser das Gleichgewicht hält. Unter diesen Umstånden war es nicht unwahrscheinlich, daß das Quellungsmaximum einer gegebenen Gelatine von dem Volumen des im Moment des Erstarrens entstandenen strukturferten Korpers abhängig sei; gewiB war dies bei folgendem Experiment der Fall.

Lösungen mit einem Gehalt von ungefabr 5, 10 und 20 Proz. lufttrockener Gelatine wurden in Glasröbren gebracht, der bequemeren Handhabung wegen auf Drahtspiralen, wurden dann einige Tage lang im trockenen Luftstrom getrocknet, dann gewoger, hernach bei Laboratoriumstemperatur in Wasser gebracht und in Intervallen immer wieder gewogen. Wenn man das Gewicht vollstăndig trockener Gelatine als Eiuheit nimmt, waren die absorbierten Wassermengen folgende :

Tabelle 1

Absorption von Wasser durch Gelatine

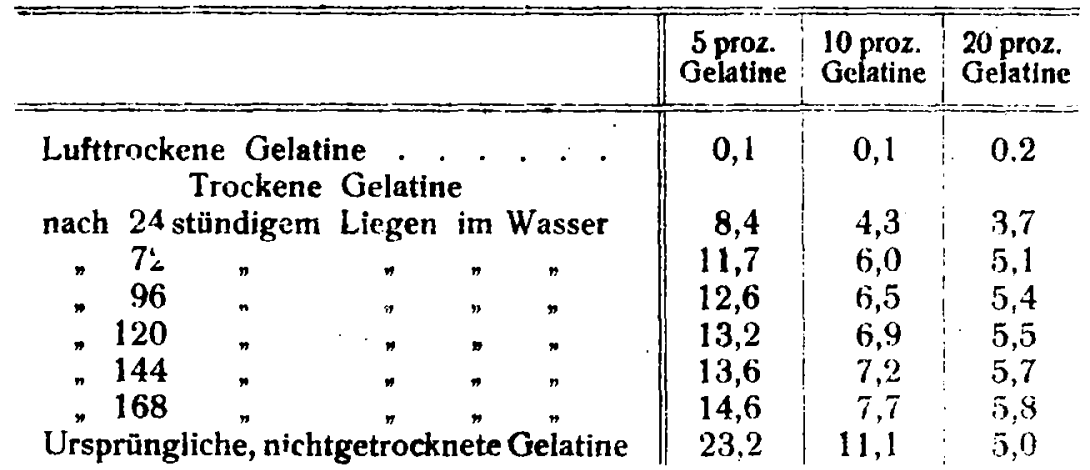


- Wie aus diesen Zahlen und noch deutlicher aus der graphischen Kurve ersichtlich ist, hat das ursprüngliche Erstarrungsvolumen bedeutenden Einflub auf die maximale Quellung, ist aber sicher nicht die einzige entscheidende Ursache.

\section{Wirkung von Alkohol auf Gelatinegallerte.}

Es ist. wohl bekannt, daB die Quellung von Gelatinegailerte durch Behandlung mit alkoholischen Losungen reduziert werden kann; in absolutem Alkohol wird die Gallerte zu einer harten und trocken aussehenden Masse. Da kein Grund besteht, eine chemische Einwirkung von Alkohol auf Gelatine anzunehmen, die beim Einwirken von Wasser wieder ihre gewöhnliche gallertartige Konsistenz annimmt, so erscheint die Alkoholwirkung günstig für das Studium der Wirkung rein physikalischer Kräfte auf Gelatine. Gelatine ist praktisch vollständig unlöslich . in absolutem oder verdünntem kalten Alkohol, und umgekehrt sind sogar ganz schwache Gallerten für alkoholische Lösungen semipermeabel. Wird Alkohol in eine poröse Zelle gebracht, die mit Gelatine belegt ist, so entwickelt er einen bedeutenden osmotischen Druck, und groBe Mengen von Gelatinegallerte, die durch Alkohol dehydratisiert werden, absorbieren kaum etwas von ihm.

Um einen Begriff von der Wirkung des Alkohols auf die Quellung zu bekommen, wurden gewogene Mengen von lufttrockener dünnplattiger Gelatine in eine Reihe von Mischungen von Wasser und Alkohol gebracht, 24 Stunden darin belassen, dann wieder für 24 Stundeh in frisch hergestellte, gleichzusammengesetzte Lösungen gebracht. Diese Zeit hatte sich in vorausgegangenen Versuchen ausreichend zur Herstellung des praktischen Gleichgewichts erwiesen. Die Gelatine wurde dann getrocknet und gewogen, um die Quellung zu bestimmen; dann wurde das Gewicht des rückstăndigen Alkohols bestimmt und sein Prozentgehalt nach den gewöhnlichen Tabellen berechnet; die Gelatinestückchen wurden in heiBem Wasser gelöst und diese Lösung destilliert, bis das Destillat $25 \mathrm{ccm}$ betrug; aus dem Gewicht des Destillates wurde der Alkoholgehalt der Gelatine bestimmt. Nur bei Verwendung von 100 prozentigem Alkohol betrug das Gewicht des Destillates 0,999 oder gar nur 0,9979 , so daB der hier nachgewiesene Alkohol sehr leicht nur der Oberfläche der Gelatine adhäriert haben kann; es müssen exaktere Arbeitsmethoden gefunden werden, damit eine Lőslichkeit des Alkohols in Gelatine nachgewiesen werden könne, obwohl es möglich erscheint, daß von nahezu dehydratisierter Gelatine etwas Alkohol aufgenommen wird. 
Es wurde noch eine zweite Serie dieser Versuche in gleicher Weise ausgeführt, bel der die Gelatine vor der Behandlung mit alkoholischen Lösungen 24 Stunden lang in Wasser quellen gelassen worden war. Die Resultate, die dann unten angeführt werden, sind nahezu vollständig mit denen der ersten Serie übereinst/minend, nur daB bei 90 und 100 Proz: Alkohol das Gleichgewicht nicht erreicht worden zu sein scheint. Es konnte kein Eindringen des Alkohols in die Gelatine nachgewiesen werden, das Gewicht des Destillates betruy zwischen 0,9996 und 1. Das Gleichgewicht scheint vollständig unikehrbar zu sein.

Tabelle 2 und Figur 1 zeigen das Gewicht der geguollenen Gelatine, bezogen auf $1 \mathrm{~g}$ Trockengewicht. Es ist zu bemerken, daß der Verlauf der Kurve ein vollständig regelmäBiger ist. Das (iewicht. der Gelatine, die in absolutem Alkohol war, ist erwas geringer als das Gewicht der nicht vorbehandelten lufttrockenen.

Tabelle 2

Wirkung der Mischungen von Alkohol und Wasser

\begin{tabular}{|c|c|c|c|c|c|}
\hline \multicolumn{2}{|c|}{$=0=-1=$} & \multicolumn{2}{|c|}{ Lufttrockene Gelatine } & \multicolumn{2}{|c|}{ Vorher gequollene Gelatine } \\
\hline \multicolumn{2}{|c|}{$\begin{array}{l}\text { Urspr. Mischung } \\
\text { von } \\
\text { absolutem“ } \\
\text { Alkohol und Wasser }\end{array}$} & $\begin{array}{l}\text { Alkohol in } \\
\text { der Lösung } \\
\text { nach dem } \\
\text { Versuch }\end{array}$ & $\begin{array}{l}\text { Gewicht von } \\
\text { I g Trocken- } \\
\text { gelatine nach } \\
\text { dem Versuch }\end{array}$ & $\begin{array}{l}\text { Alkohol in } \\
\text { der Losung } \\
\text { nach dem } \\
\text { Versuch }\end{array}$ & $\begin{array}{l}\text { Gewicht von } \\
1 \text { g Trocken- } \\
\text { gelatine nach } \\
\text { dem Versuch }\end{array}$ \\
\hline $\mathrm{ccm}$ & $\mathbf{c c m}$ & Prozenit & g & Prozent & $\mathbf{g}$ \\
\hline 100 & - & 99,04 & 1,20 & 92,63 & 1,84 \\
\hline 90 & 10 & 84,08 & 1,36 & 81,76 & 1,80 \\
\hline 80 & 20 & 73,30 & 1,50 & 70,64 & 1,60 \\
\hline 70 & 30 & $\cdots$ & - - & 60,88 & 1,68 \\
\hline 60 & 40 & 51,56 & 1,70 & 48,73 & 1,96 \\
\hline 50 & 50 & 43,04 & 2,24 & 39,80 & 2,36 \\
\hline 40 & 60 & 33,22 & 2,86 & 30,84 & 2,52 \\
\hline 30 & 70 & 24,46 & 3,14 & 22,77 & 3,06 \\
\hline 20 & 80 & 16,20 & 3,72 & 15,17 & 4,42 \\
\hline 10 & 90 & 7,93 & 5,72 & 7,42 & 5,96 \\
\hline- & 100 & - & $7,2.3$ & -- & 7.23 \\
\hline
\end{tabular}

Es ist natürlich unmöglich, den osmotischen Druck von Alkohol in so konzentrierten Lösungen, wie sie hier verwendet wurden, theoretisch zu berechnen; die Kurve ist jedoch so, wie sie zu erwarten ist, wenn ein osmotischer Druck auf eine Materie wirkt, die selbst eine gewisse Elastizität hat, und es scheint also kein Grund vorhanden 


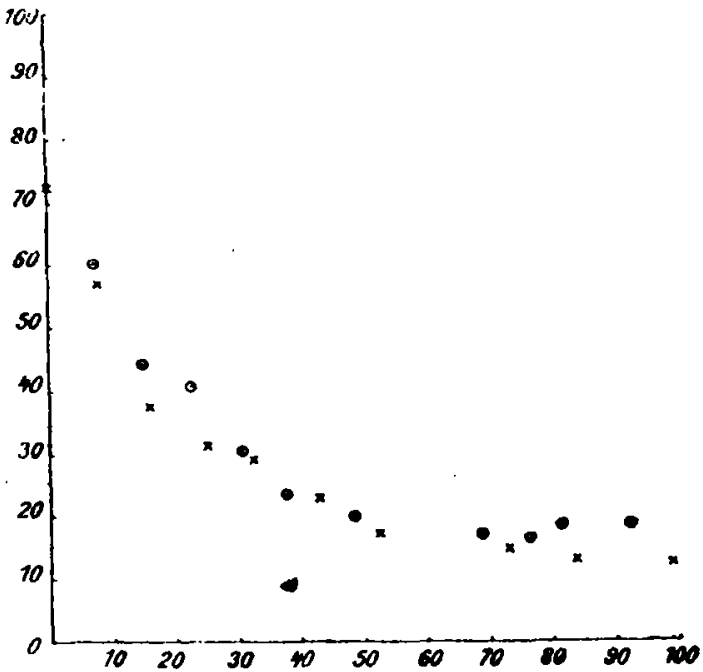

Fig. 1 (zu Tab. 2)

Ordinate:

Gewicht von $1 \mathrm{~g}$ Troekengelatine nach dem Versuch.

Abszisse :

Alkoholgehalt der Losung mach dem Versuch in Prozenten, und zwar: $x=$ Lufttrockene Gelatine; $O=$ Vorher gequollene Gelatine.

zu sein, andere Kräte zur Erklärung heranzuziehen. Wahrscheinlich ware durch unabhangige Bestimmung der osmotlschen Drucke in alkoholischen Lösungen ein Mittel gegeben, um die Elastizitàt der Gelatine zu bestimmen. Wenn man die geringe Anziehung zwischen Alkohol und Gelatine vernachlässigt, so ist das Gleichgewicht wahrscheinlich

$\left.\begin{array}{l}\text { Kohästve Affinităt der Gelatine } \\ \text { Affinltät des Alkohols für Wasser }\end{array}\right\}=\begin{gathered}\text { Affinitüt der Gelatine } \\ \text { für Wasser. }\end{gathered}$

Das ist natülich nur ein umgekehrter Weg, um das Phănomen vom osmotischen Gesichtspunkt darzustellen, da ja osmotische Drucke kinetische "Partial "drucke sind und als "negative imere Drucke" des Lösungsmittels bezeichnet werden können, geradeso wie in einer Mischung von Luft und Wasserdampf bei dem Druck einer Atmosphäre der l.uftdruck in der Mischung durch den des Wasserdampfes herabgesetzt wird, der also einem Minus an atmosphärischem Druck äquivalent ist.

Obwohl Alkohol erwärmte Gelatinelösungen präzipitiert, wenn er ihnen in groBer Menge beigefügt wird, kann er doch in geringeren 
Mengen beigemischt werden, ohne eine solche Trennung hervorzurufen, und es setzt sich dann eine scheinbar homogene Gallerte ab, die, wenn Alkohol in Gelatine unlöslich ist, tatsächlich aus einer AlkoholWasserlosung bestehen muB, deren Konzentration dem eben besprochenen Gleichgewichtszustand entspricht, und die in Form einer Emulsion in einem Gelatine-Medium eingeschlossen ist. Eine derartige Emulsion múBte in Wasser stärker quellen, als eine reine Gelatine, da nicht nur die rielatine so viel Wasser aufnehmen wird, daB sie ihr Quellungsmaximum erreicht, sondern auch die alkoholischen Emulsionspartikel verdünnt werden müssen und einen äuberlichen Druck auf die Gelatinemasse ausüben würden. Wăre die Wirkung des Alkohols auf die Gelatine, die Absorptionsfahigkeit der Gelatine zu vermindern, aber chemischer Natur, so würde die Quellung in jedem Fall verringert, ob nun der Alkohol von außen zugeführt würde, oder ob er schon in der Misching gegenwărtig wăre. Diesbezüglich wurde nun experimentell gefunden, dab von zwei ziemlich konzentrierten Gelatinen gleicher Stärke, deren eine mit Wasser allein, die andere mit einer Mischung von Wasser und Alkohol bereitet war, die letztere viel stärker quoll, was den Emulsionscharakter alkoholischer Gelatine bestätigt. Es ist nahezu sicher, daß solche Gelatine die mikroskopisch zellulare Struktur zeigen würde, die $\mathrm{O}$. Bütschli ünd $J . M$. van Bemmelen den Gelatinen im allgemeinen zuschreiben.

\section{Die Wirkung von Săuren auf Gelatine.}

Es ist wohlbekannt, daB gelatinøse Fasern durch alle verdünnten Sauren, wenn sie genügend ionisiert sind, zum Quellen gebracht werden, obwohl sehr schwache Säuren, wie Borsäure, Kohlensăure und Schwefelwasserstoff, nur eine geringe quellende Wirkung haben; dasselbe gilt für viele der schwăcheren organischen Săuren.

Die Wirkung auf Gelatine ist die gleiche. Eine Gelatine, die das Sieben- oder Achtfache ihres eigenen Gewichtes an reinem Wasser aufnimmt, kann mehr als das 50 fache ihres Gewichtes aus einer stark verdünnten Salzsăure aufnehmen. Bei unseren sehr detaillierten Versuchen wurde Salzsăure verwendet, als eine hochionisierte und typisch einbasische Slure, die sich sowohl azidimetrisch als durch Silbernitrat leicht bestimmen lieb. Ein weiterer Grund für diese Wahl war, daß, obwohl beim geworhnlich geübten Vorgang des Pickelns Schwefelsăure in Verbindung mit einem UeberschuB an Kochsalz zur 
Verwendung gelangt, die eigentlich wirksame Săure doch nur Salzsăure sein kann; und da sich durch diese und Salz allein ausreichendes Pickeln erreichen läBt, so war prinzipiell nichts dadurch zu gewinnen, daß man das Gleichgewicht durch Gegenwart von Schwefelsăure und Sulfaten gestört hätte.

Die allgerneine Versuchsmethode war der schon beschriebenen ähnlich. Stücke von lufttrockener Plattengelatine von ungefähr $1 \mathrm{~g}$ Gewicht, deren Gehalt an trockener aschenfreier Gelatine bekannt war, wurden für 48 Stunden in Săurelösungen gebracht, deren Menge und Konzentration bekannt war; diese Zeit erwies sich als ausreichend zur Erlangung eines konstanten Gleichgewichts. Menge und Stărke der rückstăndigen Lösung wurden azidimetrisch mittels gestellter $\mathrm{KOH}$ und Phenolphthalein kontrolliert; die Quellung der Gelatine wurde bestimmt, indem sie nach dem Trocknen gewogen und dann geschmolzen wurde; die absorbierte Säure wurde dann gleicherweise titriert, nachdem durch vorangegangene Experimente festgestellt worden war, daß sich derart die ganze vorhandene Săure bestimmen ließ und daB durch das Schmelzen der Gelatine das Resultat nicht verăndert wurde. Geringe Aenderungen dieser Prozedur werden bei Anführung der einzelnen Versuchsserien vermerkt.

In einer der ersten Versuchsreihen war gefunden worden, daB bei Verwendung von Phenolphthalein als Indikator die ganze Menge der in der Gelatine vorhandenen Säure, durch Methylorange aber nur ein Teil der Săure angezeigt wurde, obwohl beide Indikatoren für freie Salzsăure gleich empfindlich sind. Es ist daher klar, dab in der Gelatine ein Teil der Säure entweder chemisch oder durch Adsorption gebunden ist, derart, $\mathrm{daB}$ er weniger $\mathrm{H}$-Ionen abdissoziiert als der Rest, der sich verhält. als ob er nur in der Gelatine gelōst wăre und natürlich mit dem Grad der Quellung variiert. Um die so eingeführte Komplikation ausschalten zu können, wurde vorerst angenommen, dab -die aufgenommene Säure dieselbe Konzentration habe, wie die umgebende Saurelösung, und daß der UeberschuB, der bei Titration mit Phenolphthalein immer gefunden wurde, an der Gelatine "fixiert" oder jedenfalls enger mit dieser verbunden sei. Diese ,fixierte Säure * erwies sich gewöhnlich als etwas weniger konzentriert, aber an Menge ungefähr der entsprechend, die wohl durch Phenolphthalein, nicht aber durch Methylorange nachweisbar war und stellt offenbar den durch die Gelatine aufgenommenen Săureüberschuß dar, obwohl es sich nicht genau bestimmen läbt, welche Menge der Gelatine anhaftet und welche dem absorbierten Wasser; späler wird noch gezeigt werden, 
daB die tatsächlich durch die Gelatine absorbierte Säuremenge gröBer ist, als die so nachgewiesene. Dennoch bietet die Kenntnis der so bestimmiten „fixierten Säure" ein geeignetes Mittel, den Charakter der Absorptionen vergleichen zu können, ınd deshalb ist sie in den Tabellen angeführt.

Tabelle 3

Gelatine und Salzsäure

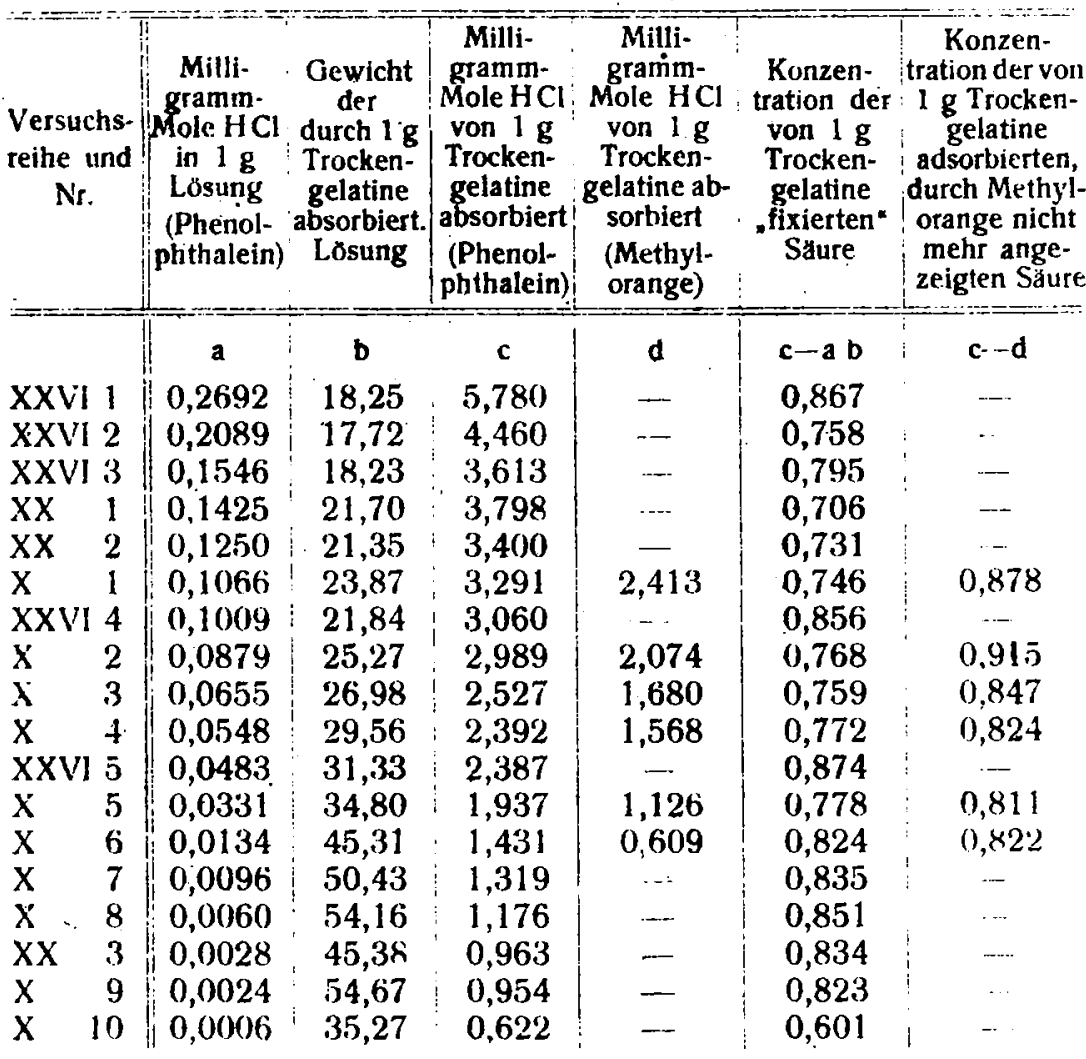

Tab. 3(vgl. Fig. 2 )fabt Resultate aus mehreren Versuchsreihen zusammen (die römischen Ziffern in der ersten Kolonne zeigen die Versuchsreihe an). Diese Experimente wurden gröBtenteils in den Jahren 1899 und 1900 an einem Muster französischer Gelatine ausgeführt, die meisten Titrationen machte damals Herr R. Poget. Tab. 4 (vgl. Fig. 2) bringt Versuche, die elst kürzlich ausgeführt wurden, mit geringen Aenderungen der Methode, wie sie die Erfahrung gelehrt hatte; die dabei verwendete Gelatine hatte mehr Säurecharakteı und scheinbar eine stärkere K()- 


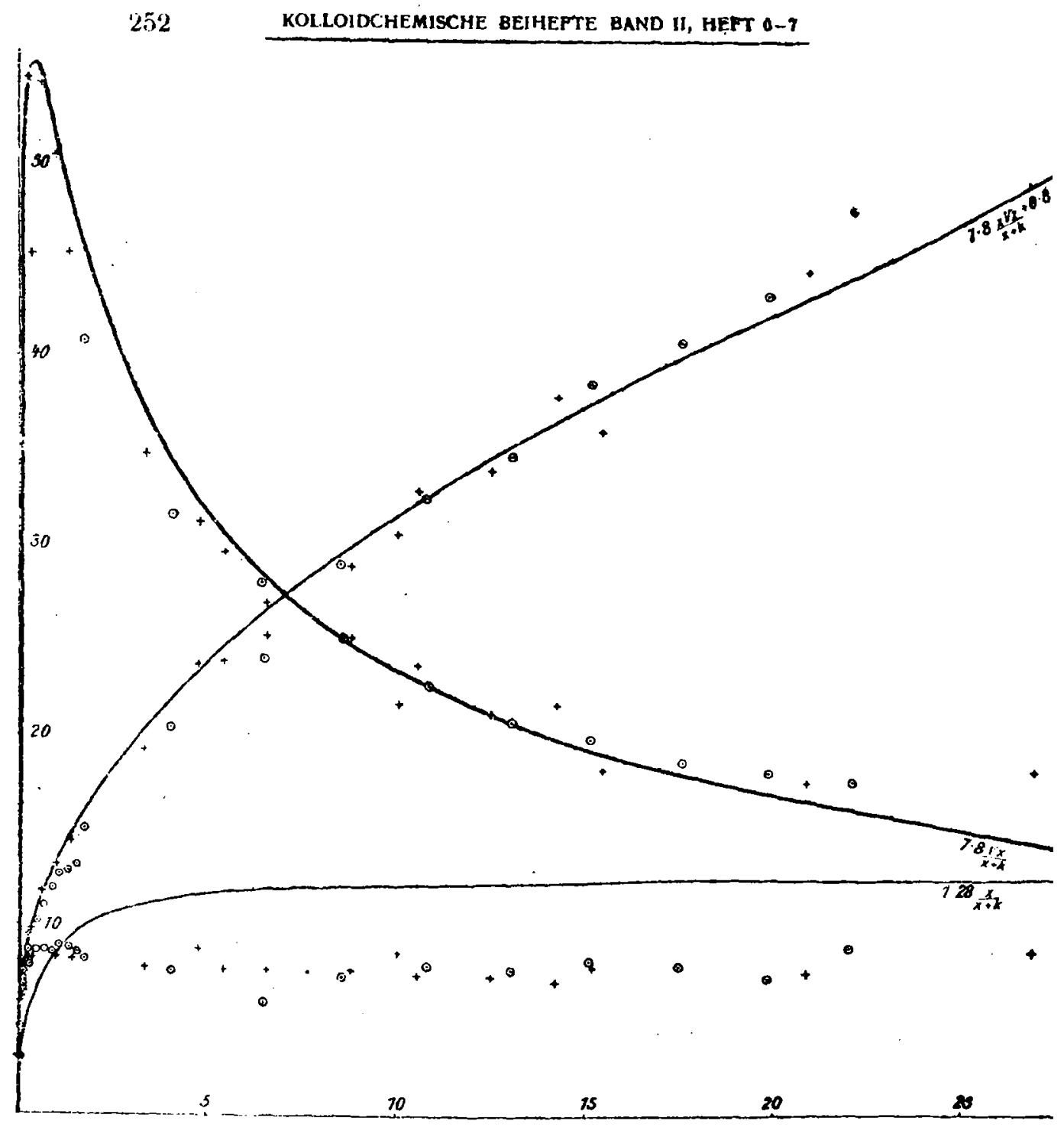

Fig. 2 (zu Tab. 3 und 4)

Abszisse: $\mathrm{Mg}$-Mole $\mathrm{HCl}$ in $1 \mathrm{~g}$ Losung.

Ordinate: $\frac{1}{+}=$ Resultate aus Tab. $3 ; 0=$ Resultate aus Tab. 4.

häsion, da die maximale Quellung in allen Fallen geringer ist, obwohl der Charakter der Kurven und die Lage der Maxima im allgemeinen gute Uebereinstimmung mit den früheren Resultaten zeigt. Besonders bei den schwächeren Konzentrationen in Tabelle 4 wird durch die sauren Figenschaften der Gelatine eine gewisse $Z$ Weideutigkeit ver- 


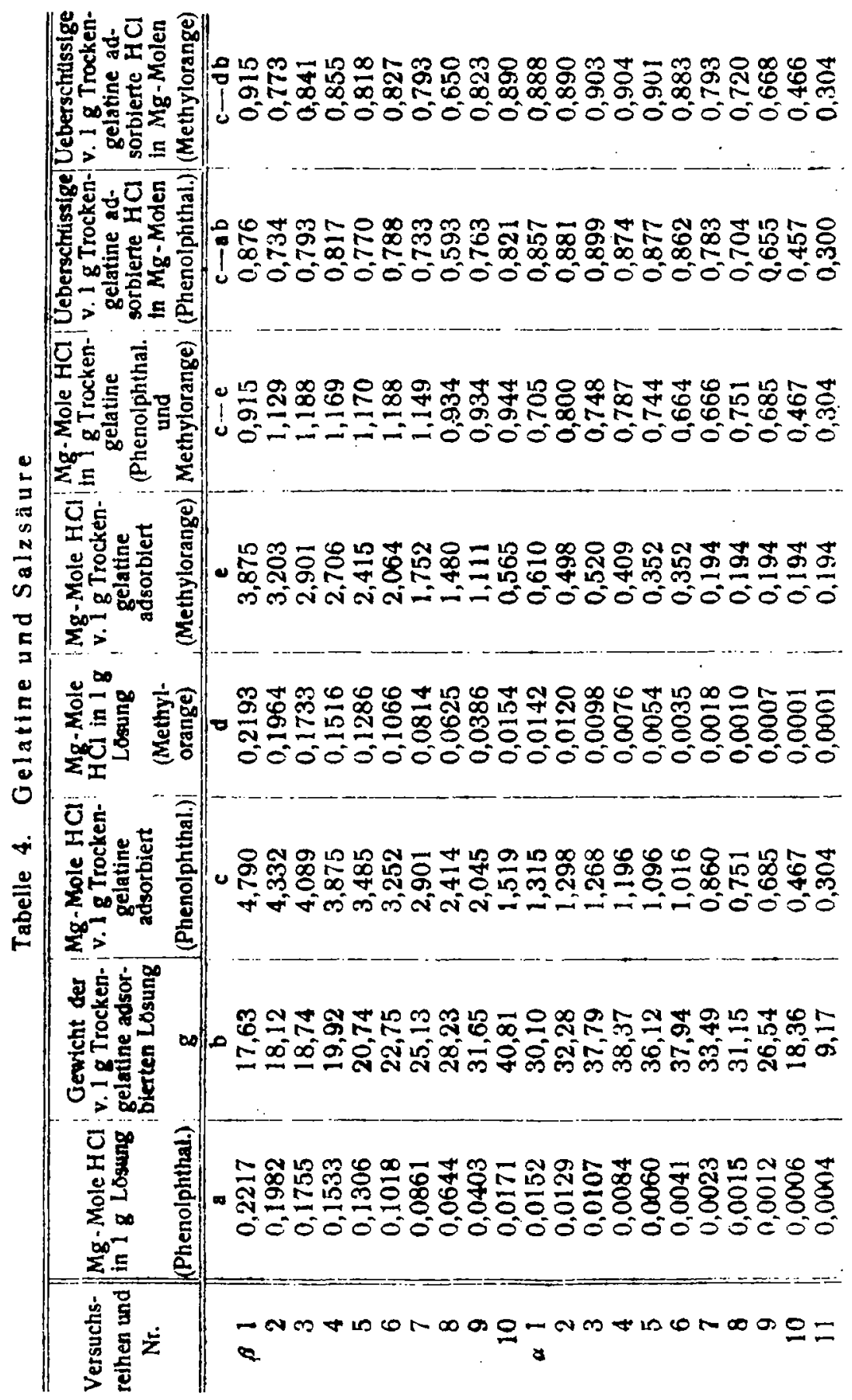


ursacht: diese Săuerung der Gelatine ist hauptsächlich auf die darin vorhandenen Bisulfate zurückzuführen, deren Menge bis zu 0,282 Milligrammole pro Gramm anstieg, und die durch Phenolphthalein, nicht aber durch Methylorange angezeigt wurden; von ihnen diffundierte zumindest ein Teil in die äubere Lōsung und becinflubte deren aus Phenolphthaleintitrationen berechneten molekularen Săuregehalt; aber auf die "fixierte" Saure konnten sie wohl schwerlich groben EinfluB haben. Es wurden deshalb Bestimmungen der Kqnzentration der äußeren Lösung mittels Methylorange gemacht, die gewiß die ganze in Form von $\mathrm{HCl}$ vorhandene Säure angibt, und dann wurde mit Hilfe beider Indikatoren die "fixierte "Säure und die durch die Gelatine absorbierte Säure berechnet.

Bei Besprechung dieser Untersuchungen muB auch einer Arbeit von Wo. Ostwald') über die Quellung der Gelatine Erwähnung getan werden, in der er die Quellung von Gelatineplatten in Săuren und Alkalien verschiedener Konzentrationen bestimmt. In beiden Fallen konnte er das Vorhandensein eines Maximums, wie es eben beschrieben wurde, nachweisen; er beobachtete aber bei Anwendung von sehr verdünnten Lösungen auch ein Minimum, von dem in diesen Untersuchungen keine Spar gefunden werden konnte. Wo. Ostwald selbst schreibt dies ebenfalls der ursprünglich sauren Reaktion der Gelatine zu, und das ist wahrscheinlich korrekt, da die sauren Eigenschaften gewöhnlich von Bisulfaten herrühren, die starken Basen gegenüber sauer, starken Säuren gegenüber alkalisch sind, und deren Neutralisation in beiden Fällen wahrscheinlich die ursprüngliche Quellung in Wasser vermindern würde. Die von ihm verwendeten Platten waren viel dicker $(3.4 \mathrm{~mm}$ im Vergleich zu ungefahr $0,25 \mathrm{~mm})$ als die in meinen Versuchen verwendeten, so daß es zweifelhaft ist; ob überhaupt ein vollkommenes Gleichgewicht in seinen Versuchen erreicht wurde.

In Tabelle 5 sind die Resultate einer Versuchsserie enthalten, die ausgeführt wurde, um die Umkehrbarkeit des Gleichgewichts Gelatine -..Säure---Wasser zu prüfen. Die Gelatine wurde 48 Stunden ing in einer Losung aufquellen gelassen, deren Konzentration, wenn das Gleichgewicht erreicht war, 0,2253 Millimole betrug, dann für 24 Stunden in lösungen von verschiedener (geringerer) Konzentration gebracht. Diese zweite Zeit scheint nicht vollkommen ausreichend

1) Wo. Ostwald, , Ueber den Einfluß von Săuren und Alkalien auf die Quellung der Gelatine. Archiv fuir die ges. Physiol. 108 (1905). 
zur Wiedererlangung des Gleichgewichts gewesen zu sein, doch besteht kein Grund, anzunehmen, daß die Adsorption nicht vollkommen umkehrbar sei. Die geringe Konzentration der „fixierten Säure“, die berechnet wurde, indem man die als adsorbiert berechnete Säure von der absorbiert gefundenen . abzieht, ist einigermaßen bemerkenswert, und es ist bedauerlich, dab in dieser Serie keine Parallelbestimmungen mit Methylorange gemacht wurden. Es wären ja verschiedene Erklärungen mōglich, aber es wird wohl am besten sein, die Diskussion aufzuschieben, bis weitere Untersuchungen vorliegen. Die Proben, die nur 48 Stunden mit einer Lösung behandelt wurden, sind in bezug auf "fixierte " Săure normal.

Tabelle 5

Gelatine, die 48 Stunden hindurch in $\mathrm{HCl}$ von ungefähr 0,275 Milligrammols pro Gramm quellen gelassen worden war; Nr. 4--16 kamen hierauf für 24 Stụnden in schwächere Lösung.

\begin{tabular}{|c|c|c|c|c|}
\hline $\begin{array}{c}\text { Versuchs- } \\
\text { reihe } \\
\text { Nr. }\end{array}$ & $\begin{array}{l}\mathrm{Mg} \text {-Mole } \mathrm{HCl} \\
\text { in } 1 \mathrm{~g} \text { Losung } \\
\text { (Phenolphthal.) }\end{array}$ & $\begin{array}{l}\text { Gewicht } \\
\text { der von.1 g } \\
\text { Trockengelatine } \\
\text { absorb. Losung }\end{array}$ & $\begin{array}{l}\mathrm{Mg} \text {-Mole } \mathrm{HCl} \\
\text { von } 1 \mathrm{~g} \text { Trocken- } \\
\text { gelatine absorb. } \\
\text { (Phenolphíhal.) }\end{array}$ & $\begin{array}{c}\text { Konzentration } \\
\text { der von } 1 \mathbf{g} \\
\text { Trockengelatine } \\
\text { fixierten" Săure }\end{array}$ \\
\hline & $+\mathbf{a}$ & b & c & $c-a b=f$ \\
\hline $\begin{array}{r}1 \\
2 \\
3\end{array}$ & $\begin{array}{l}0,2253 \\
0,2253 \\
0,2253\end{array}$ & $\begin{array}{l}20,33 \\
20,14 \\
21,18\end{array}$ & $\begin{array}{l}5,411 \\
5,411 \\
5,506\end{array}$ & $\begin{array}{l}0,830 \\
0,873 \\
0,734\end{array}$ \\
\hline
\end{tabular}

Darauf 24 Stunden in schwächeren Lösungen

\begin{tabular}{r||l|l|l|l}
4 & $\mathbf{0 , 2 3 3 3}$ & 22,00 & $\mathbf{5 , 6 8 3}$ & 0,541 \\
$\mathbf{5}$ & $\mathbf{0 , 2 1 4 6}$ & 21,61 & $\mathbf{5 , 1 2 5}$ & $\mathbf{0 , 4 8 8}$ \\
$\mathbf{6}$ & 0,1950 & 21,10 & 4,616 & $\mathbf{0 , 5 0 2}$ \\
7 & $\mathbf{0 , 1 7 5 4}$ & 22,77 & 4,567 & 0,573 \\
$\mathbf{8}$ & $\mathbf{0 , 1 5 6 1}$ & 23,44 & 4,247 & 0,615 \\
$\mathbf{9}$ & $\mathbf{0 , 1 3 8 3}$ & 25,04 & 4,039 & 0,576 \\
10 & $\mathbf{0 , 1 1 8 2}$ & 24,37 & $\mathbf{3 , 5 3 1}$ & 0,650 \\
11 & 0,0984 & 26,89 & 3,218 & 0,571 \\
12 & 0,0807 & 27,90 & 2,944 & 0,692 \\
13 & 0,0629 & 28,94 & 2,465 & 0,644 \\
14 & 0,0526 & 29,33 & 2,220 & 0,673 \\
15 & 0,0476 & 32,87 & 2,230 & 0,667 \\
16 & 0,0462 & 31,18 & 2,083 & 0,643
\end{tabular}

Obwohl von dieser etwas rohen Versuchsmethode keine exakten quantitativen Resultate zu erwarten waren, ist es klar, daß die graphischen Darstellungen der Versuche aus den Tabellen 3 und 4, in Fig. 2 , 
die Kurven eines regelmäBigen Gleichgewichts darstellen, das für die verschiedenen Serien der Untersuchung nahezu gleich bleibt; die experimentellen Fehler sind nicht gröBer, als es zu erwarten war, wenn man den Einflub verschiedener nicht bestimmter Faktoren in Betracht zieht, wie die Kohäsionselastizităt der Gelatine, und die außerordentlich geringen Krăfte, die in der Nähe des Quellungsmaximums bereits als bemerkenswerte Volumsănderungen zum Ausdruck kommen. Der nach einer bestimmten Konzentration annähernd horizontal verbleibende Verlauf der Kurve der "fixierten Săure“ legt den Gedanken sehr nahe, daB es sich eher um einen bestimmten, obwohl stets hydrolysierenden chemischen, als um einen wirklichen physikalischen Komplex kandelt; ferner weisen die Bestimmungen der "fixierten Săure" aus der Differenz der Reaktionsänderungen des Phenolphthaleins und Methylorange auf einen deutlichen Unterschied der $\mathrm{H}$-Ionen-Konzentrationen in der Nähe des betrefienden Punktes hin. Dafür ist das sehr deutliche Maximum des Quellungsvolumens (S) bewejsend, und dieses folgt natürlich aus der Kombination der zwel gewőhnlichen Kurven, der "Gesamtsäure" pro Gramm Gelatine (a) und der ,fixierten Saure ${ }^{\text {( }}$ ( $)$ entsprechend $\operatorname{der}$ Formel $S=a, f$, wobei $x$ die Konzentration der äußeren Lösung bedeutet, entsprechend der Kolumne a der Tabellen.

Ueber die anderen Săuren liegen nur wenige Versuche vor. Mit schwachen Săuren, z. B. Essig- und Milchsăure war kein distinktes Quellungsmaximum zu beobachten, die Adsorption der Flüssigkeit nahm eben mit der Konzentration zu, bis die Lösung der Gelatine begann. Die Ameisensăure zeigte ein Maximum bei einer Konzentration von zirka 0,07 Grammolen im Liter, aber es ist weniger deutlich, und der Anstieg zum Maximum ist vor allem viel allmählicher als bei der Salzsäure. Da schwächere Säuren also kein Zurückgehen der Quellung bewirken, kann man eine gröbere totale Quellung erreichen, wenn man die Lősung mit Essig- und vielleicht auch mit Milchsăure konzentriert, als wenn man es mit Salzsăure tut, obwohl in dem letzteren Fall ja ganz sicher eine stärkere Auflösung und vielleicht auch Strukturveranderungen der Gelatine eintreten.

Die Fixation der Säure kann man mittels der Methylurangemethode nur bei den stärksten Säuren untersuchen, wenn man aber die adsorbierte Losung als gleich konzentriert mit der äuberen ansieht, dảnn erhălt man fär die Adsorption Figuren, die für Essigsäure von mittlerer Konzentration etwas niedriger und füı Milch- und Amıeisen- 
säure ungefähr gleich oder eine Spur höher sind, als bei der Salzsäure. In höheren Konzentrationen ist die gesamte adsorbierte Săuremenge so groß, daß experimentelle Fehler die Bestimmung der fxierten Säure undeutlich und unregelmåBig machen, und die Werte steigen von den niedrigsten Konzentrationen zu einem bestimmten Mittelwert viel allmählicher als bei den starken Säuren.

Ueber die Schwefelsăure liegen nur wenige Bestimnungen vor, die alie darauf hinweisen, daf sie in einer niedrigen Konzentration ein Quellungsmaximum bildet, das noch nicht genau bestimmt wurde und die "fixierte Säuremenge" ist gleichfalls etwas größler. Zur Bestimmung der "fixierten Saure" sind Methylorangeversuche nicht gemacht worden; da der Farbwechsel bei diesem Indikator aber sehr allmahlich ist, so sind wohl Untersuchungen durch bloße Titration ziemlich grob, und wir haben uns vorgenommen, dieser Frage mittels der Untersuchung der lonisationskonstanten näher zu kommen.

Die folgenden Tabellen 6-9 enthalten die Resultate der Versuche mit den eben besprochenen Săuren.

Tabelle 6

Gelatine und Essigsäure

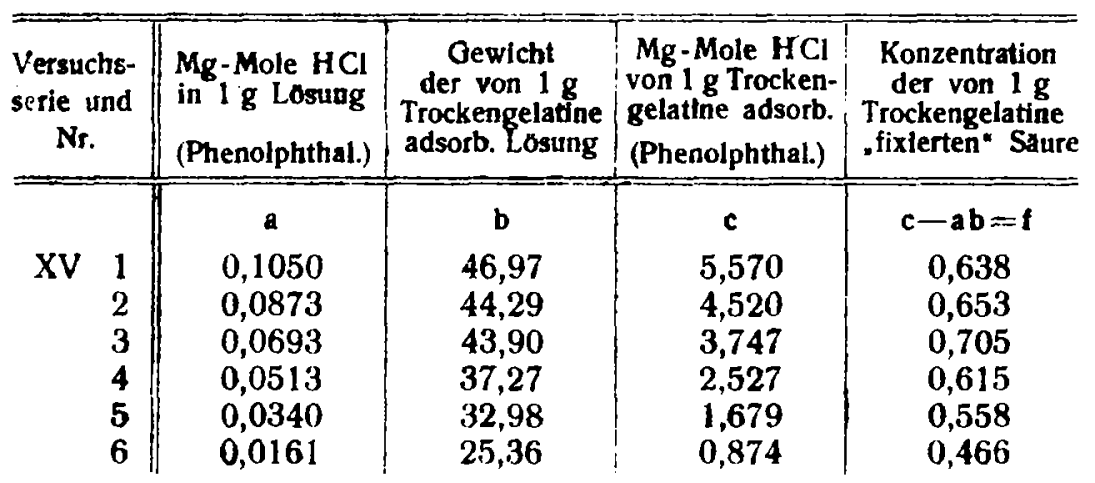

Andere Gelathesorte :

\begin{tabular}{cc||c|c|c|c}
$\mathrm{A}$ & 1 & 1,0050 & 62,97 & 62,54 & 0,74 \\
& 2 & 0,5083 & 57,28 & 32,05 & 2,93 \\
& 3 & 0,2062 & 51,14 & 11,50 & 0,95 \\
4 & 0,1011 & 44,68 & 5,127 & 0,61 \\
& 5 & 0,0805 & 33,89 & 3,398 & $\mathbf{0 , 6 7}$ \\
& 6 & 0,0512 & 28,81 & 2,042 & $\mathbf{0 , 3 9 5}$ \\
7 & 0,0216 & 20,56 & 0,917 & $\mathbf{0 , 4 7 3}$ \\
8 & 0,0110 & 17,35 & 0,954 & $\mathbf{0 , 4 0 ?}$
\end{tabular}


Tabelle 7

Gelatine und Milchsäure

\begin{tabular}{|c|c|c|c|c|}
\hline $\begin{array}{l}\text { Versuchs- } \\
\text { serie und } \\
\text { Nr. }\end{array}$ & $\begin{array}{l}\mathrm{Mg} \text {-Mole } \mathrm{HCl} \\
\text { in } 1 \mathrm{~g} \text { Losung } \\
\text { (Phenolphthal.) }\end{array}$ & $\begin{array}{c}\text { Gewicht } \\
\text { der von } 1 \mathrm{~g} \\
\text { Trockengelatine } \\
\text { adsorb. Losung }\end{array}$ & $\begin{array}{l}\mathrm{Mg} \text {-Mole } \mathrm{HCl} \\
\text { von } 1 \mathrm{~g} \text { Trocken- } \\
\text { gelatine adsorb. } \\
\text { (Phenolphthal.) }\end{array}$ & 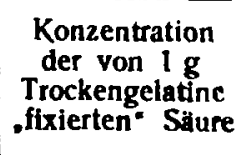 \\
\hline & $\mathbf{a}$ & b & c & $c-a b=f$ \\
\hline $\begin{array}{l}1 \\
2 \\
3 \\
4\end{array}$ & $\begin{array}{l}0,1047 \\
0,0684 \\
0,0321 \\
0,0048\end{array}$ & $\begin{array}{l}52,73 \\
48,63 \\
48,19 \\
26,98\end{array}$ & $\begin{array}{l}6,284 \\
4,124 \\
2,397 \\
0,673\end{array}$ & $\begin{array}{l}0,764 \\
0,798 \\
0,825 \\
0,54: 3\end{array}$ \\
\hline
\end{tabular}

Tabelle 8

Gelatine (andere Sorte) und Ameisensäure

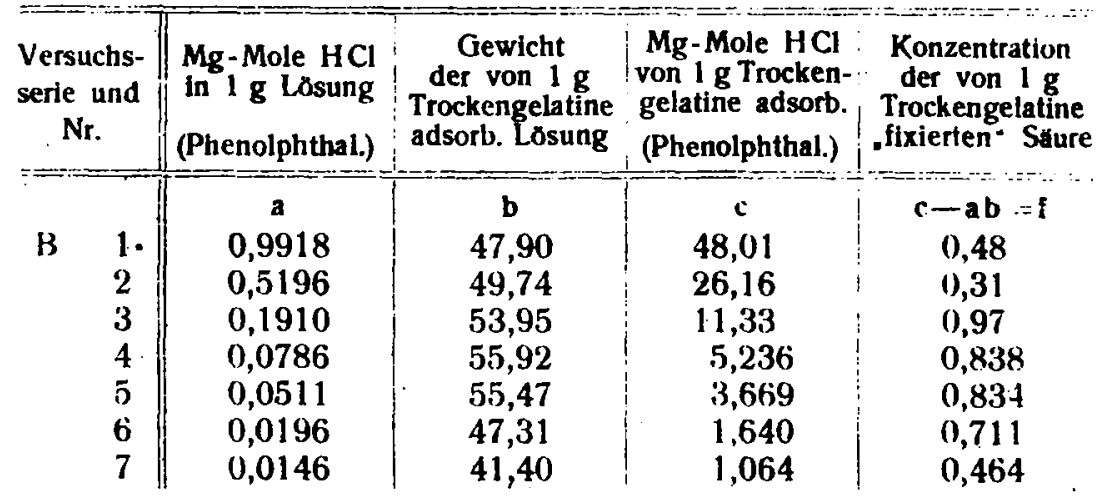

Tabelle 9

Gelatine und Schwefelsaure

\begin{tabular}{|c|c|c|c|c|}
\hline $\begin{array}{l}\text { Versuchs- } \\
\text { serie und } \\
\text { Nr. }\end{array}$ & {$\left[\begin{array}{c}1 / 2 \mathrm{Mg} \text {-Mole } \mathrm{HCl} \\
\text { in } 1 \text { gLo sung } \\
\text { (Phienolphthal.) }\end{array}\right.$} & $\begin{array}{c}\text { Gewicht } \\
\text { der von } 1 \mathrm{~g} \\
\text { Trockengelatine } \\
\text { adsorb. Losung }\end{array}$ & $\begin{array}{l}1 / 2 \mathrm{Mg}-\text { Mole } \mathrm{HCl} \\
\text { von } 1 \text { g Trocken- } \\
\text { gelatine adsorb. } \\
\text { (Phienolphthal.) }\end{array}$ & $\begin{array}{l}\text { Konzentration } \\
\text { der von } 18 \\
\text { Trockengelatine } \\
\text { fixierten* Săure }\end{array}$ \\
\hline $\begin{array}{ll}\text { XVI } & 1 \\
& 2 \\
& 3 \\
& 4\end{array}$ & $\begin{array}{c}\mathrm{a} \\
0,1055 \\
0,0685 \\
0,0303 \\
0,0024\end{array}$ & $\begin{array}{c}\text { b } \\
20,17 \\
20,85 \\
25,27 \\
31,65\end{array}$ & $\begin{array}{c}c \\
3,178 \\
2,469 \\
1,855 \\
0,944\end{array}$ & $\begin{array}{c}c-a b=f \\
1,050 \\
1,041 \\
1,089 \\
0,868\end{array}$ \\
\hline
\end{tabular}

In Tabelle 10 (XXIX, 1--3) sind einige wenige Untersuchungen an der Schafhaut registriert, aus denen hervorgeht, dab auch hier ein Quellungsmaximum besteht, indem schwăchere Lōsungen mehr quellend wirken als stärkere und daß die Menge der "fixierten Säure* 
ungefähr dieselbe ist wie bei der Gelatine. Die Haut war auf die gewöhnliche Weise enthaart worden, entkalkt, bei $80^{\circ}$ getrocknet und ins Wasser getaucht, bis sie weich wurde.

Tabelle 10

Schaffell, bei $80^{\circ} \mathrm{C}$ getrocknet, und Salzsäure

\begin{tabular}{|c|c|c|c|c|c|c|}
\hline \multirow{2}{*}{$\begin{array}{c}\text { Ver- } \\
\text { suchs- } \\
\text { serie u. } \\
\text { Nr. }\end{array}$} & \multicolumn{2}{|c|}{ Originallosung } & \multirow{2}{*}{$\begin{array}{c}\mathrm{Mg}-\text { Mole } \\
\mathrm{HCl} \text { in } \\
\text { der } \\
\text { Losung }\end{array}$} & \multirow{2}{*}{$\begin{array}{c}\text { Gewicht } \\
\text { der von der } \\
\text { Haut ad- } \\
\text { sorbierten } \\
\text { Losung }\end{array}$} & \multirow{2}{*}{$\begin{array}{l}\mathrm{Mg} \text {-Mole } \\
\mathrm{HCl} \text { von } \\
\mathrm{lg} \text { Haut } \\
\text { adsorbiert }\end{array}$} & \multirow{2}{*}{$\begin{array}{l}\text { Konzen- } \\
\text { tration der } \\
\text { v. } 1 \mathrm{~g} \text { Haut } \\
\text { fixierten } \\
\text { Saure }\end{array}$} \\
\hline & $\begin{array}{c}\text { Mg-Mole } \\
\mathrm{HCl} \text { in } \\
1 \mathrm{~g} \text { Lösung }\end{array}$ & $\begin{array}{c}\mathrm{Mg}-\text { Mole. } \\
\mathrm{NaCl} \text { in } \\
1 \mathrm{~g} \mathrm{Losung}\end{array}$ & & & & \\
\hline & & & a & b & c & $c-a b=-f$ \\
\hline XXIX 1 & $\begin{array}{l}0,1466 \\
0,0488 \\
0,0073\end{array}$ & $\begin{array}{l}-- \\
- \\
-\end{array}$ & $\begin{array}{l}0,1386 \\
0,0466 \\
0,0024\end{array}$ & $\begin{array}{l}10,38 \\
10,76 \\
19,31\end{array}$ & $\begin{array}{l}2,176 \\
1,236 \\
0,467 .\end{array}$ & $\begin{array}{l}0,737 \\
0,735 \\
0,421\end{array}$ \\
\hline $\begin{array}{r}X \times X \\
-\quad 2 \\
3\end{array}$ & $\begin{array}{l}0,1807 \\
0,0907 \\
0,0378\end{array}$ & $\begin{array}{l}3,00 \\
3,01 \\
3,02\end{array}$ & $\begin{array}{c}0,1665 \\
0,0798 \\
\ldots\end{array}$ & $\begin{array}{l}5,95 \\
5,70 \\
4,71\end{array}$ & $\begin{array}{l}2,389 \\
1,499 \\
0,364\end{array}$ & $\begin{array}{l}1,399 \\
1,044 \\
0,364\end{array}$ \\
\hline $\begin{array}{r}\text { XXXI } 1 \\
2 \\
3\end{array}$ & $\begin{array}{c}0,0692 \\
0,0826 \\
0,0981\end{array}$ & $\begin{array}{l}4,17 \\
1,66 \\
0,48\end{array}$ & $\begin{array}{l}0,0580 \\
0,0700 \\
0,0775\end{array}$ & $\begin{array}{l}4,11 \\
4,27 \\
3,93\end{array}$ & $\begin{array}{l}1,455 \\
1,315 \\
1,227\end{array}$ & $\begin{array}{l}1,217 \\
1,016 \\
0,922\end{array}$ \\
\hline
\end{tabular}

Hier ist es am Platze, auf eine Untersuchung von E. Stiasny ${ }^{\text {, }}$ einzugehen, der mittels ganz anderer'Methoden, als die hier von mir verwendeten, über die Adsorption von Wasser und Săuren durch Hautpulver und BlőBe arbeitete. Aus den dort angeführten Daten ist es wohl sehr schwer oder ganz unmöglich, die Menge „fixierter Săure " zu berechnen, aber in beiden Fällen tritt sehr deutlich ein Quellungsmaximum in schwächeren Konzentrationen auf. Die Resultate der Versuche mit Hautpulver und Blöße zeigen beträchtliche Unterschiede, die E. Stias ny auf den Unterschied im Gewebe zurückführt, deren Grund aber vielleicht zum Teil darin liegt, daß die Zeit. zur Einstellung eines vollständigen Gleichgewichtes ungenügend war.

Eine der auffallendsten Tatsachen beim "Pickeln", die ja auch den Anstob zu diesen Untersuchungen gab, ist die auBerordentliche Dehydratation, die stark konzentrierte Lösungen der gewöhnlichen Salze auf die angesäuerten Hautfasern ausüben ebenso wie auf die angesäuerte Gelatine; angesäuerte Gelatíne wird aus ihren warmen Lösungen

1) E. Stiasny, Ueber negative Adsorption und die Bestimmung der Schwellwirkung von Săuren auf Hautpulver und BloBe. Gerber $183 \mathrm{ff}$. (1909) und Collegium 302 ff. (1909). 
durch sie sogar als zusammenhăngende Masse gefallt. Durch die gewöhnlichen Salze allein, also an neutralen Hautfasern oder Gelatine, kommen solche Wirkungen nicht zustande, da ja auch gesättigte Salzlösungen die Quellung ein wentg erhöhen: Eine Gelatine. die ungefähr das Achtfache ihres Gewichtes un Wasser adsorbiert, nimmt ungefähr das Elffache aus einer gesăitigten Salzlösung und eine noch grobBere Menge Wasser aus einer Salzlosung mittlerer Konzentration auf. Einige Reihen diesbezüglicher Experimente sind in Tabelle 11 verzeichnet. Einige andere Salze hingegen, besonders Ammoniumsulfat und andere Sulfate, üben, wie man ja längst weiß, eine mächtige dehydrierende Wirkung auf gequollene Gelatine oder auf Haut aus und vermögen sogar Gelatine aus ihren warmen Lösungen als kohărente Masse auszufällen. Mit einer Diskussion dieser Wirkungen der Neutralsalze muß man wohl warten, bis die Wirkungen der Salze in sauren Lösungen besser studiert sein werden; trotzdem aber mußk ich darauf hinweisen, daß die Sulfate der schwachen Basen, besonders Ammonium und Zink, am stärksten wirken.

Tabclle 11

Gelatine und verschieden konzentrierte Salzlösungen

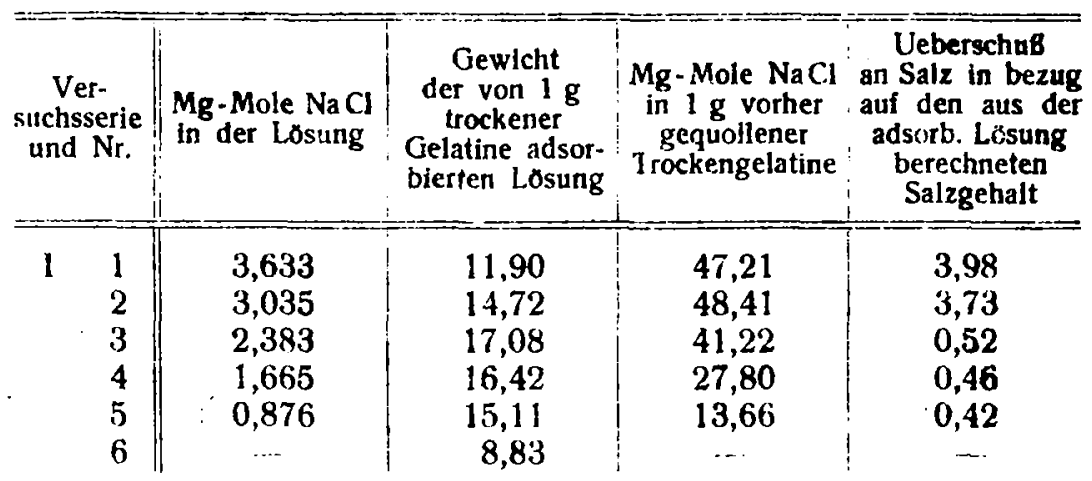

Es ist gleichgültig, ob die Gelatine oder die Hautfaser zuerst in der Säure quellen gelassen und dann der Salzwirkung ausgesetzt werden, oder ob der Vorgang umgekehrt stattfindet, denn man erhalt die gleichen Resultate, wenn man Săure in entsprechender Konzentration zu der Salzgelatine hinzusetzt, und man kann auch pickeln, wenn man eine bestimmte Säuremenge zu den Häuten zusetzt, die in einem stark gesalzenen Wasser liegen, obwohl diese letztere Methode kostspieliger ist. Die wirksamste Säurekonzentration ist größer, als die zur Herbeiführung des Quellungsmaximums nöttg ist, da die 
Gegenwart von Salzen die Haut oder die Gelatine befähigt, eine größere Säuremenge zu "fixieren“, als sie es in der gleich starken Säurelossung ohne Salz imstande wäre. In Gegenwart von genügend Salz kann man aber auch - und in der Industrie geschieht das gewöhnlich so - einen großen Säureüberschub verwenden, ohne daß die Resultate merklich beeinflubt werden; doch ist ein groBer Ueberschub unerwünscht, denn er vermindert in verdünnten Salzlosungen die Dehydratation. Einige Wirkungen von angesäuerten Salzlösungen auf Haute sind in Tabelle 10 zusammengefabt und die Resultate einer großen Zahl von Experimenten über den Einfluß von Salzsăure und Salzen in verschiedenen Konzentrationen auf Gelatine in Tabelle 12 u. 13.

Wenn man diese Resultate mit denen der Tabellen 3-5 vergleicht, in welchen die Resultate mit Salzsăure allein verzeichnet sind, so făllt auf, daß die von $1 \mathrm{~g}$ Gelatine adsorbierte Gesamtsäure geringer ist, indem durch die Volumskontraktion, die durch das Salz verursacht wird, ebenso Săure wie Wasser herausgepreBt wird. Andererseits aber ist die "fixierte Säure", die berechnet wurde, indem man von der gesamten adsorbierten Säure die in dem gleichen Volumen einer wässerigen Lösung enthaltene abzog, auf alle Fälle größer als bei der Salzsăure allein. Daraus kann man aber nicht unbedingt schließen, daß die von der Gelatine wirklich adsorbierte Säuremenge größer ist, wenn Salze zugegen sind, wohl aber, daß die Dissoziation oder Hydrolyse geringer ist. Andererseits kann man daraus aber auch nicht folgern, daB die jedenfalls unbewiesene Annahme unrichtig ist, die Säurekonzentration der adsorbierten Lösung sei der der uußeren gleich. Wenn aber, wie es wahrscheinlich ist, die Konzentration der adsorbierten Lösung wirklich geringer ist, dann wird die ,fixierte Säure “ eben zunehmen, wenn die Menge der adsorbierten Lossung sbnimmt.

Andererseits aber muB man auch hervorheben, daB die minder ionisierte Säure, wie aus ihrer Unwirksamkeit gegenüber dem Methylorange hervorgeht, relativ und sogar auch absolut weniger ionisiert ist" in der salzhaltigen, als in der blob sauren salzfreien Lösung. Es ist aber auch keineswegs wahrscheinlich, daß das Methylorange vollständig unempfindlich gegenüber der Azidität der Säuregelatine ist; wenn das aber nicht der Fall ist, dann wird die Wirkung der Säuregelatine um so größer sein, je größer die Săurekonzentration in der kontrahierten Gelatine ist, sowie ja das Methylorange durch viele organische Säuren gerötet wird, wenn sie in konzentrierten Lösuagen vorhanden sind, aber nur sehr wenig in verdünnten. Jede direkte Einwirkung der Säuregelatine anf Methylorange wird die Menge 


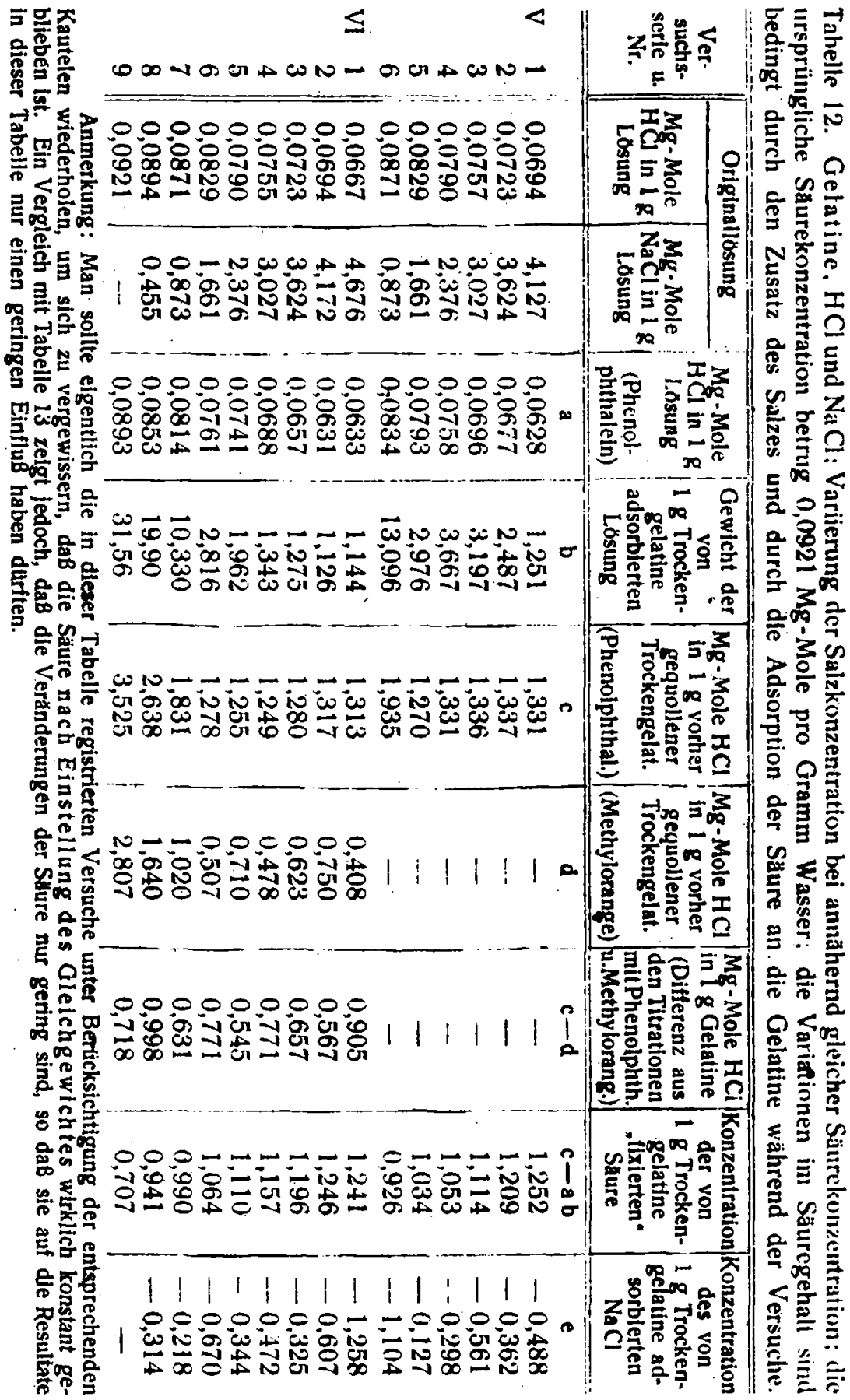


Tabelle 13. Gelatine, $\mathrm{HCl}$ und $\mathrm{NaCl}$. Variation der Säurekonzentration bei annăhernd gleichen Salzkonzentrationen; die Săurekoqzentration wurde nach Einstellung des Gleichgewichts bestimmt, die Salzkonzentration als Verdünnung der Ausgangslösung berechnet, da ja ihre Adsorption negativ ist und die adsorbierte Menge im Vergleich zur Gesamtkonzentration vernachlässigt werden kann.

\begin{tabular}{|c|c|c|c|c|c|c|c|}
\hline $\begin{array}{l}\text { Ver- } \\
\text { suchs- } \\
\text { serie } \\
\text { and } \\
\text { Nr. }\end{array}$ & $\begin{array}{l}\text { Mg-Mole } \\
\text { MCl in 1 } \\
\text { Losung } \\
\text { (Phenol- } \\
\text { phthaleta) }\end{array}$ & $\begin{array}{l}\text { Oewicht } \\
\text { der von } 1 \mathrm{~g} \\
\text { Trocken-: } \\
\text { gelatipe ad- } \\
\text { oolbienten } \\
\text { Losung }\end{array}$ & $\begin{array}{l}\text { Mg-Mole } \\
\text { HC1 in } 1 \mathrm{~g} \\
\text { Trockeen- } \\
\text { gelatine } \\
\text { (Phenol- } \\
\text { phthalein) }\end{array}$ & $\begin{array}{l}\text { Mg- Mole } \\
\text { Hel in } 1 \mathrm{~g} \\
\text { Trocken- } \\
\text { gelatine } \\
\text { (Methyl- } \\
\text { orange) }\end{array}$ & $\begin{array}{c}\text { Mg-Mole } \\
\text { HCl in } 1 \mathrm{~g} \\
\text { Trockengelat. } \\
\text { (Difierenz aus } \\
\text { denTritrationen } \\
\text { mit Phenol- } \\
\text { phthalein nnd } \\
\text { Methylorange) }\end{array}$ & $\begin{array}{l}\text { Konzen- } \\
\text { tration der } \\
\text { yon 1 } 1 \\
\text { Trocken- } \\
\text { gelatine } \\
\text { "fixlerten " } \\
\text { Siure }\end{array}$ & $\begin{array}{l}\text { Kouzen- } \\
\text { tration des } \\
\text { von 1 g } \\
\text { Trocken- } \\
\text { gelatine } \\
\text { fixierten" } \\
\text { Salzes }\end{array}$ \\
\hline & $\mathbf{a}$ & b & c & d & $c-d$ & $c-a b$ & \\
\hline 1 & 0,0832 & 1,57 & 1,239 & 0,229 & 1,108 & 1,108 & $+0,784$ \\
\hline 2 & 0,0702 & 1,52 & 1,228 & 0,567 & 0,661 & 1,121 & 0,064 \\
\hline 3 & 0,0601 & $1 ; 3$ & 1,257 . & 0,554 & 0,703 & 1,175 & $+0,354$ \\
\hline 4 & 0,0410 & $1,64^{\circ}$ & 1,199 & 0,514 & 0,685 & 1,132 & $-0,329$ \\
\hline 5 & 0,0251 & 2,05 & 1,138 & 0,438 & 0,700 & 1,087 & $-0,545$ \\
\hline 6 & 0,0126 & 2,0 & & & & & $-0,762$ \\
\hline VII 1 & 0,1796 & 1,54 & 1,508 & 0,592 & 0,916 & 1,232 & $-0,912$ \\
\hline 2 & 0,1429 & 1,58 & 1,434 & 0,548 & 0,886 & 1,198 & $-0,853$ \\
\hline 3 & 0,1184 & 1,52 & 1,381 & 0,565 & 0,816 & 1,201 & $-0,885$ \\
\hline 4 & 0,0872 & $1,60^{\circ}$ & 1,303 & 0,6 & 0,638 & 1,163 & $-0,620$ \\
\hline 5 & 0,0717 & 1,58 & 1,287 & 0,539 & 0,748 & 1,174 & $-0,495$ \\
\hline 6 & 0,0564 & 1,58 & 1,248 & 0,414 & 0,861 & 1,159 & $-0,731$ \\
\hline & 0,0409 & 1,50 & 1,225 & 0,4 & 0,805 & 1,164 & $-0,690$ \\
\hline 8 & 0,0262 & 1,50 & 1,179 & 0,288 & 0,891 & 1,140 & $-0,629$ \\
\hline 9 & 0,0100 & 1,57 & 1,118 & 0,259 & & 1,102 & - \\
\hline 10 & 0,0021 & 1,62 & 1,095 & 0,209 & 0,886 & 1,091 & $-0,735$ \\
\hline 11 & 0,0002 & 3,16 & 0,728 & - & 0,727 & 0,727 & $--0,516$ \\
\hline 12 & -. & 15,78 & $\left.0,109^{n}\right)$ & $\ldots$ & 0,109 & 0,109 & $+0,180$ \\
\hline
\end{tabular}

Ursprüngliche Konzentration der Salzlösung 3,02 Mole pro Gramm

\begin{tabular}{|c|c|c|c|c|c|c|c|}
\hline VIII 1 & 0,1817 & 13,62 & 3,495 & 2,673 & 0,822 & 1,020 & $\cdots$ \\
\hline & 0,1465 & 12,98 & 3,028 & 1,977 & 1,051 & 1,127 & $\cdots$ \\
\hline & 0,1154 & 12,57 & 2,553 & 1,558 & 0,995 & 1,102 & - \\
\hline & 0,0861 & 11,63 & 2,088 & 1,093 & 0,995 & 1,087 & - \\
\hline & 0,0722 & 10,93 & 1,842 & 0,855 & 0,987 & 1,052 & ... \\
\hline & 0,0570 & 11,73 & 1,698 & 0,871 & 0,827 & 1,029 & -. \\
\hline & 0,0347 & 11,52 & 1,426 & 0,508 & 0,918 & 1,026 & \\
\hline 8 & 0,0247 & 10,99 & 1,295 & 0,465 & 0,830 & 1,024 & $\ldots$ \\
\hline $\mathbf{9}$ & 0,0096 & 10,73 & 1,142 & 0,221 & 0,921 & 1,039 & $\ldots$ \\
\hline 10 & 0,0023 & 9,81 & 1,010 & 0,147 & 0,863 & 0,987 & - \\
\hline 11 & 0,0002 & 10,44 & 0,684 & - & 0,684 & 0,682 & -. \\
\hline 12 & - & 11,90 & 0,103 & $\ldots$ & 0,103 & 0,103 & - \\
\hline
\end{tabular}

Ursprïngliche Konzentration der Salzlősungen 0,76 Mole pro Gramm

1) Diese Azidităt ist die Eigenazidităt der Gelatine; sie ist hauptsachlich auf Bisulfate zuridckzufuhren. 
fixierter-Săure, die auf die angegebene Weise berechnet wurde, vermindern.

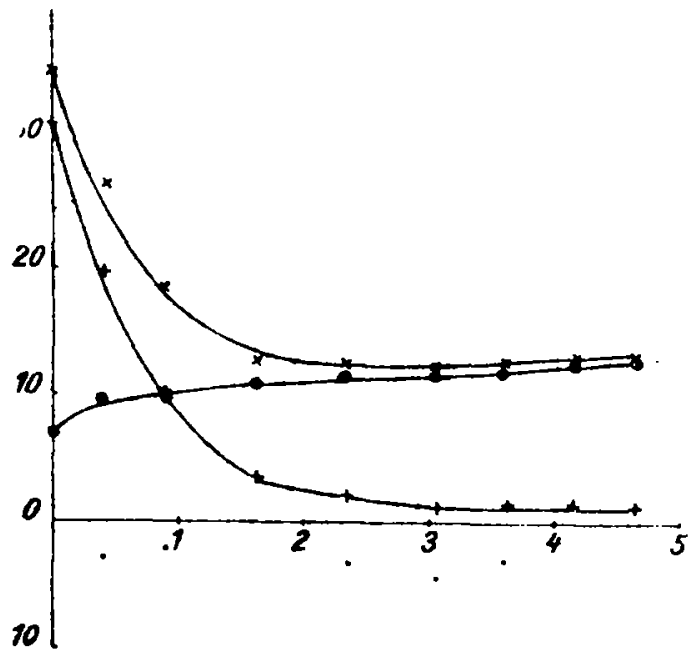

Fig. 3 (zu Tab. 12)

Abszisse:

$\mathrm{Mg}$-Mole $\mathrm{NaCl}$ in $\mathrm{I} \mathrm{g}$ losung.

Ordinate:

$x=\mathrm{Mg}$-Mole $\mathrm{HCl}$ in $1 \mathrm{~g}$ Trockengelatine; $\mathrm{O}=$ Konzentration der von 1 g Trockengelattine, firjerten" Slure; $t=$ Gewicht der von

$1 \mathrm{~g}$ trockengelatine adsorbierten Losung; . = ,fixiertes" Salz.

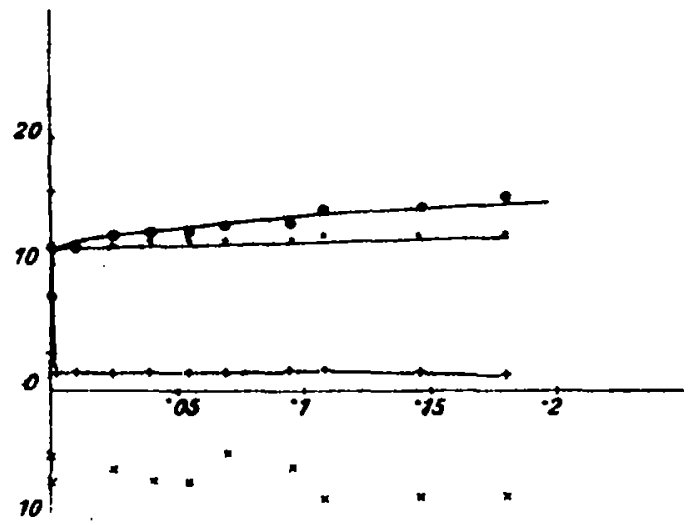

Fig. 1 (zu Tab. 13, Serie VII)

Abszlsse: $\mathrm{Mg}$-Mole $\mathrm{HCl}$ in $1 \mathrm{~g}$ Losung (a).

Ordinate:

$O=\mathrm{Mg}$-Mole $\mathrm{HCl}$ in $1 \mathrm{~g}$ Trockengelatine; $+=$ Gewicht der von

l g Trockengelatine adsorbierten Lơsung (b);.$=$ „fixlerte* Sắufe; $x=$.fixiertes" Salz. 
Interessant ist die Tatsache, die aus den Tabellen 12 und 13 hervorgeht, betreffs der Konzentration des "fixierten“ Salzes; dessen Adsorption erscheint numlich in Gegenwart von Salzsäure immer negativ; die wenigen Ausnahmen sind wohl sicher auf experimentelle Fehler zurückzuführen. Aus den Versuchen in Tabelle 11 ersieht man aber auch, dab die Konzentration des an die Gelatine "fixierten* Salzes in Abwesenheif von Săuren stets positiv "ist. Die Mengen wurden auf folgende Weise berechnet: Man bestimmt den gesamten Chlorgehalt in der Gelatine mittels Silbernitrat und Kaliumchromat und subtrahiert davon die azidimetrisch bestimmte Salzsäuremenge und den gesamten berechneten Chlorgehalt der adsorbierten Lösung. Infolge der großen Gesamtkonzentration der anwesenden Chloride und der verhaltnismabig großen Wirkung des adsorbierten Teils der Lösung kann man für den Verlauf dieser Versuche keine sehr große quantitative Genauigkeit verlangen; immerhin legt er uns aber die Annahme sehr nahe, daB die durch die Gelatine als Salzsăure adsorbierten Chloride auf die Dissoziation der Chlorionen (der Säure und des Salzes) in der Lösung eine entsprechende vermindernde Wirkung ausüben. Es wurde im Zusammenhang mit den Experimenten erwăhnt, dab in einzelnen Fällen, wo große Salzmengen verwendet wurden, die Gelatinestückchen weiß und opak wurden anstatt homogen und transparent; das mub man wohl entweder der Kristallisation von Salz in der Gelatine zuschreiben oder aber der Bildung zellularer oder zumindest heterogener Strukturen, die Losungen einschlieBen, die einen anderen Brechungsindex haben, als die umgebende Gelatine. Es ist überaus wahrscheinlich, daB dieser Umstand die Ursache der Unregelmäigkeiten im Verlauf der Fixation der Salze ist.

Aus den Kurven in Figur 4 geht hervor, daß der Verlauf der Quellungsabnahme einer Hyperbel entspricht, die mit zunehmender Salzkonzentration sich asymptotisch einem Werte nähert, der etwas über Null liegt.

Es ist ganz klar, daB jede Säure mit ihren entsprechenden Neutralsalzen auf die gequollene Gelatine eine dehydrierende Wirkung ausübt, deren Intensităt mit der elektrolytischen Dissoziationskonstante der Săure variiert und mit der Salzkonzentration zunimmt. Die Wirkung von Natriumsulfat in Verbindung mit Schwefelsăure ist allem Anscheine nach ebenso groB, wie die des Natriumchlorids mit Salzsăure in aquivalenten Konzentrationen, wăhrend schwache Säuren mit ihren Neutralsalzen viel weniger wirksam sind, obwohl der Effekt auch da sehr deutlich ist, wenn man ihn mit der quellenden Wirkung der 
reinen Säuren vergleicht, wie sie in Tabelle 15 und 16 resp. 6 und 8 verzeichnet sind.

Tabelle 14

Schwefelsäure und Natriumsulfat

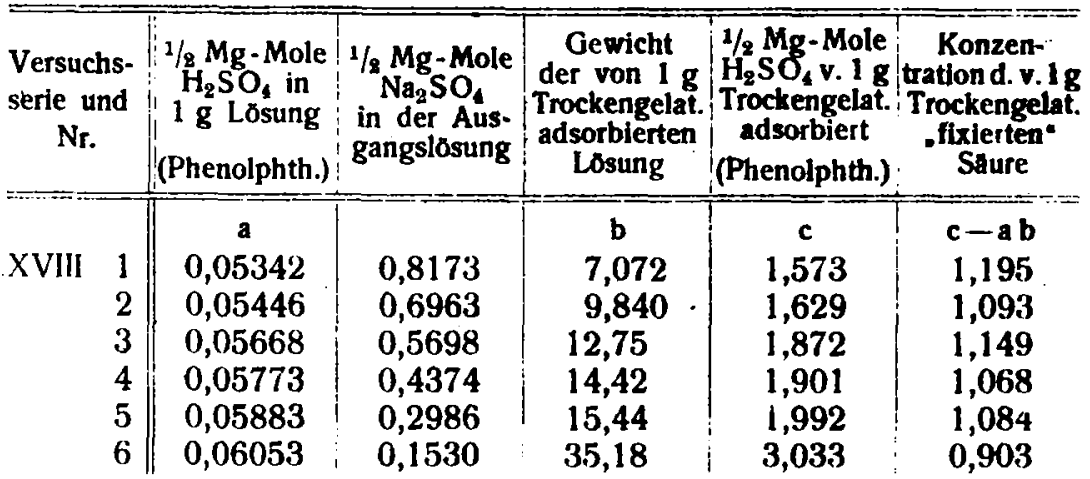

Bei den schwächeren Săuren ist der UeberschuB von adsorbierter Säure gegenüber der aus der Menge der adsorbierten Losung berechneten, also die „wirklich fixierte Säure*, viel geringer als bei den stärkeren und sehr unregelmäBig; dies gilt vor allem fur die schwach ionisierte Essigsăure, deren Ionisation durch die gleichzeitige Anwesenheit der Azetate noch reduziert wird.

Tabelle 15

Gelatine, Ameisensăure und Natriumformiat (1,33 n Natriumformiatlosung)

\begin{tabular}{|c|c|c|c|c|}
\hline $\begin{array}{l}\text { Versuchs- } \\
\text { serie und } \\
\text { Nr. }\end{array}$ & $\begin{array}{l}\text { Mg-Mole Săure } \\
\text { in } 1 \mathrm{~g} \text { Losung }\end{array}$ & $\begin{array}{l}\text { Gewicht } \\
\text { der von } 1 \mathrm{~g} \\
\text { Trockengelatine } \\
\text { adsorb. Losung }\end{array}$ & $\begin{array}{c}\mathrm{Mg} \text { - Mole Sture } \\
\text { von } 1 \mathrm{~g} \\
\text { Trockengelatine } \\
\text { adsorbiert }\end{array}$ & $\begin{array}{l}\text { Konzentration } \\
\text { der von } 1 \mathrm{~g} \\
\text { Trockengelatine } \\
\text { fixierten" Sture }\end{array}$ \\
\hline \multirow{9}{*}{$\mathrm{D}$} & $\mathbf{a}$ & $\mathbf{b}$ & c & $c-\mathbf{a} \mathbf{b}$ \\
\hline & 0,9008 & 19,45 & 18,39 & 0,87 \\
\hline & 0,4955 & 17,14 & 9,41 & 0,62 \\
\hline & 0,2049 & 15,53 & 3,79 & 0,61 \\
\hline & 0,1053 & 16,04 & 2,15 & 0,46 \\
\hline & 0,0824 & 16,99 & 1,71 & 0,31 \\
\hline & 0,0574 & 15,57 & 1,23 & 0,34 \\
\hline & 0,0265 & 17,07 & 0,70 & 0,25 \\
\hline & 0,0172 & 15,93 & 0,44 & 0,17 \\
\hline
\end{tabular}


Tabelle 16 Gelatine, Essigsaure und Natriumazetat (1,33 n Natriumazetat)

\begin{tabular}{|c|c|c|c|c|}
\hline $\begin{array}{l}\text { Versuchs- } \\
\text { serie und } \\
\text { Nr. }\end{array}$ & $\begin{array}{l}\mathrm{Mg} \text {-Mole Săure } \\
\text { in } 1 \mathrm{~g} \text { Losung }\end{array}$ & $\begin{array}{l}\text { Gewicht } \\
\text { der von } 1 \mathrm{~g} \\
\text { Trockengelatine } \\
\text { adsorb. Losung }\end{array}$ & $\begin{array}{l}\text { Mg - Mole Săure } \\
\text { von } 1 \mathrm{~g} \\
\text { Trockengelatine } \\
\text { adsorbiert }\end{array}$ & $\begin{array}{l}\text { Konzentration } \\
\text { der von } 1 \mathrm{~g} \\
\text { Trockengelatine } \\
\text { fixierten Säure }\end{array}$ \\
\hline & $\mathbf{a}$ & b & c & $c-a b$ \\
\hline B & 0,9656 & 20,83 & 20,64 & 0,50 \\
\hline 2 & $(0,5018$ & 17,64 & 9,74 & 0,89 \\
\hline 3 & 0,1991 & 15,89 & 3,32 & 0,16 \\
\hline 4 & 0,1007 & 14,38 & 1,56 & 0,11 \\
\hline 5 & 0,0788 & 14,20 & 1,26 & 0,14 \\
\hline 6 & 0,0526 & 13,20 & 0,79 & 0.10 \\
\hline 7 & 0,0242 & 12,26 & 0,39 & 0,09 \\
\hline 8 & 0,0143 & 11,05 & 0,18 & 0,02 \\
\hline
\end{tabular}

Tabelle 17 Gelatine, Salzsaure und Natriumsulfat

\begin{tabular}{|c|c|c|c|c|c|c|}
\hline $\begin{array}{l}\text { Ver- } \\
\text { suchs- } \\
\text { serie } \\
\text { und } \\
\mathrm{Nr} \text {. }\end{array}$ & $\begin{array}{l}\text { Mg-Mol. } \\
\text { Saure in } \\
1 \text { g Aus- } \\
\text { gangs- } \\
\text { losung }\end{array}$ & $\begin{array}{l}1 / 9 \text { Mg-Mole } \\
\mathrm{Na}_{2} \mathrm{SO} \\
\text { in } 1 \mathrm{~g} \text { Aus- } \\
\text { gangs- } \\
\text { lösung }\end{array}$ & $\begin{array}{c}\text { Mg-Mole } \\
\text { Săure in } \\
1 \mathrm{~g} \text { Losung } \\
\text { (Phenol- } \\
\text { phthalein) }\end{array}$ & $\begin{array}{c}\text { Gewicht } \\
\text { der von } 1 \mathrm{~g} \\
\text { Trockengelat. } \\
\text { adsorbjerten } \\
\text { Losung }\end{array}$ & $\begin{array}{c}\text { Mg-Molc } \\
\text { Saure von } 1 \mathrm{~g} \\
\text { Trockengelat. } \\
\text { adsorbiert } \\
\text { (Phenolphth.) }\end{array}$ & $\begin{array}{l}\text { Konzentration } \\
\text { der von } 1 \mathrm{~g} \\
\text { Trockengelat. } \\
\text { "fixierten " } \\
\text { Säure }\end{array}$ \\
\hline & & & $\mathbf{a}$ & b & c & $c-a b$ \\
\hline XII & 0,0574 & 0,8172 & 0,0560 & 7,41 & 1,492 & 1,067 \\
\hline 2 & 0,0587 & 0,6963 & 0,0524 & 8,79 & 1,457 & 0,941 \\
\hline 3 & $(0,0601$ & 0,5698 & 0,0533 & 8,07 & 1,513 & 1,028 \\
\hline 4 & $\| 0,0615$ & 0,4374 & 0,0558 & 9,24 & 1,603 & 1,035 \\
\hline 5 & 0,0630 & 0,2986 & 0,0555 & 10,03 & 1,682 & 1,050 \\
\hline b! & 0.0645 & 0,1530 & 0,0565 & 11,85 & 1,766 & 0,937 \\
\hline
\end{tabular}

Die Konzentration der Ausgangslösung der Sãure in Wasser betrug nur 0,0663 Mole.

Tabelle 18 Gelatine, Essigsāure und Kochsalz

\begin{tabular}{|c|c|c|c|c|c|c|}
\hline \multirow[t]{2}{*}{$\begin{array}{c}\text { Ver- } \\
\text { suchs- } \\
\text { serie } \\
\text { und } \\
\mathrm{Nr} \text {. }\end{array}$} & $\begin{array}{c}\text { Mg-Mole } \\
\text { Saure in } \\
1 \text { g Aus- } \\
\text { gangs- } \\
\text { losung }\end{array}$ & $\begin{array}{l}\text { Mg-Mole } \\
\mathrm{NaCl} \text { in } \\
1 \mathrm{~g} \mathrm{Aus-} \\
\text { gangs- } \\
\text { losung }\end{array}$ & $\begin{array}{l}\text { Mg-Mole } \\
\text { Saure in } \\
1 \text { g Losung } \\
\text { (Phenol- } \\
\text { phthalein) }\end{array}$ & $\begin{array}{c}\text { Gewicht } \\
\text { der von } 1 \text { g } \\
\text { Trockengelat. } \\
\text { adsorbierten } \\
\text { Losung }\end{array}$ & $\begin{array}{c}\text { Mg - Mole } \\
\text { Săurc von } 1 \mathrm{~g} \\
\text { Trockengelat. } \\
\text { adsorbiert } \\
\text { (Phenolphth.) }\end{array}$ & $\begin{array}{l}\text { Konzentration } \\
\text { der von 1 g } \\
\text { Trockengelat. } \\
\text { "fixierten } \\
\text { Säure }\end{array}$ \\
\hline & & & $\mathbf{a}$ & b & c & $c-a b$ \\
\hline$I X$ & 0,0696 & & 06 & 1,37 & 1,166 & 1,078 \\
\hline 2 & 0,0793 & 2,371 & 0,0739 & 2,65 & 1,217 & 1,021 \\
\hline 3 & 0,0874 & 0,871 & 0,0839 & 10,49 & 1,798 & 0,918 \\
\hline
\end{tabular}

Die Konzèntration der Ausgangslösung der Säure in Wasser betrug nur 0,0926 Mole. 
Tabelle 19

Gelatine, Salzsăure und Kaliumchlorid

\begin{tabular}{|c|c|c|c|c|c|c|}
\hline $\begin{array}{c}\text { Ver- } \\
\text { suchs- } \\
\text { serie } \\
\text { 'und } \\
\text { Nr. }\end{array}$ & $\begin{array}{c}\text { Mg-Mole } \\
\text { Săure in } \\
1 \mathrm{~g} \text { Aus- } \\
\text { gangs- } \\
\text { Posung }\end{array}$ & $\begin{array}{l}\mathrm{Mg} \text {-Mole } \\
\mathrm{KCl} \text { in } \\
\mathrm{I} \mathrm{g} \text { Aus- } \\
\text { gangs- } \\
\text { losung }\end{array}$ & $\begin{array}{l}\text { Mg-Mole } \\
\text { Săure in } \\
\text { lg Losung } \\
\text { (Phenol- } \\
\text { phthalein) }\end{array}$ & $\begin{array}{l}\text { Gewicht } \\
\text { der von } \mathbf{1} \mathbf{g} \\
\text { Trockengelat. } \\
\text { adsorbierten } \\
\text { Losung }\end{array}$ & $\begin{array}{c}\text { Mg - Mole } \\
\text { Saure von l g } \\
\text { Trockengelatt } \\
\text { adsorbiert } \\
\text { (Phenolphth.) }\end{array}$ & $\begin{array}{l}\text { Konzenuration } \\
\text { der von I g } \\
\text { Trockengelat } \\
\text { fixierten } \\
\text { Säure }\end{array}$ \\
\hline & & & $\mathbf{a}$ & b & c & \\
\hline & & & 0,0524 & & & \\
\hline 2 & & & 177 & & & \\
\hline 3 & & & & & & \\
\hline 4 & & & & & & \\
\hline 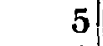 & & & & & & \\
\hline 6 & 0,0640 & 0,432 & 0,0592 & 12,26 & 1,746 & 1,020 \\
\hline
\end{tabular}

Die Konzentration der Ausgangslösung der Săure in Wasser betrug nur 0,0663 Mole.

Um eine derartige Entquellung durch Saize hervorzurufen, müBte das Salz keineswegs das gleiche Anion haben wie die Săure, und wenn das Salz einer starken Săure und in genügender Konzentration vorhanden ist, kann man eine gute Entquellung auch durch Ansäuerung mit sehr schwachen Săuren erhalten. So kann man auch mit Ameisenoder Essigsäure in Gegenwart von Neutralsalzen gut pickeln, und wenn ich mich recht erinnere, ist auch in der Industrie Ameisensăure in gewissem Ausmaß zum Pickeln der Schafhăute verwendet worden, zuma! die antiseptische Wirkung der Ameisensäure größer ist, als die der gewőhnlich verwendeten Schwefelsăure, und auch einige schädigende Wirkungen der letzteren auf das Endprodukt so vermieden werden. Gewöhnlich wird in der Industrie die Schwefelsảure mit Neutralsalzen verwendet, aber sofern man auf die Kosten keine Rücksicht nehmen will, kơnnte man ebensogut Salzsăure und Natriumsulfat verwenden, wie aus Tabelle 17 hervorgeht. In Tabelle 18 sind Versuche mit Essigsăure und Neutralsalzen registriert. Auch hier ist die Dehydratation und die Menge der fixierten Säure praktisch gleich wie bei Verwendung der Salzsăure.

In Tabelle 19 sind Versuche verzeichnet, in denen Kaliumchlorid an Stelle von Natriumchlorid, und zwar mit Salzsăure, verwendet wurde. Die Resultate sind in beiden Fallen fast identisch.

Zus ammenfassung: Gelatine adsorbiert Wasser unter Wärme entwicklung und vermag dabel anfangs einen großen Druck auszuúben; je grðBer aber das Quantum des aufgenommenen Wassers wird, um 
so mehr nimmt die Affinităt der Gelatine für das Wasser ab; man merkt dann keine Wärmeentwicklung mehr, und die wirksame mechanische Kraft ist überaus klein und betrăgt nur einige Dynen pro Sekundenzentimeter. Der Prozeß ist vollständig reversibel, und das Wasser kann ebensoleicht aus der vollstăndig gequollenen Gelatine durch mechanischen Druck herausgepreBt und durch Verdampfen im Vaku um vollstăndig entfernt werden; zum größten Teil vermögen dies auch dehydrierende Agenzien; die letzten Wasserreste lassen sich aber nur mit größter Schwierigkeit entfernen. In kaltem Wasser geht die Quellung nicht ad infinitum vor sich, sondern bis zu einem Maximum, das den Gleichgewichtszustand zwischen der Affinität der Gelatine für das Wasser und den elastischen Kohäsionskrăften der Gelatine darstellt; dieses Maximum hängt nicht nur vom chemischen Charakter, sondern auch vom ursprünglichen Erstarrungsvolumen der Gelatine ab. In heißem Wasser kommt es schlieBlich zu einer vollständigen Auflosung.

In Alkohol ist die Gelatine unloslich und die in Wasser gequollene Gelatine ist für konzentrierten Alkohol undurchlässig, der sie dehydriert. Wenn man Alkohol in die flüssige Gelatine bringt und diese dann sich absetzen låkt, so liegt das Quellungsmaximum dieser von Alkohol gefällten Gelatine in Wasser höher als das der gewöhnlichen.

Gelatine quillt schon in sehr verdünnten Säuren viel stärker als in Wasser. In schwachen Săuren nimmt diese Quellung mit zunehmender Säurekonzentration stetig $z u$, bis schlieBlich eine vollstăndige Auflösung stattfindet; in starken Săuren aber kommt es zu einem Quellungsmaximum in, sehr verdünnten Konzentrationen, darauf nimmt dann die Quellung -- und zwar in Form einer Hyperbel -wieder ab, bis sich schlieBlich die Gelatine aufløst, ohne weiter zu quellen, wodurch das Bereich des Experiments abgegrenzt ist. Fügt man jedoch zu dieser Săure noch ein Neutralsalz zu, dann vermag die Dehydratation - wenn die Săure stark ist - so weit getrieben zu werden, dab die Gelatine das Aussehen von festen hornartigen Massen annimmt. Mit schwachen Săuren und ihren Salzen ist die Wirkung geringer, aber. immerhin sehr deutlich wahmehmbar. Kombiniert man eine schwache Säure mit einem Ueberschub eines Salzes einer starken Săure, dann verhălt sich die Gelatine so, als hătte man eine starke Säure angewendet; läbt man die Gelatine aber in einer starken Säure quellen, und behandelt sie dann mit dem Salz einer schwachen Săure, dann verhält sie sich so, als wäre nur eine schwache Säure wirksam. 
Dic von der gequollenen Gelatine adsorbierte Säuremenge Ist inımer größer, als die in der Gelatine befindliche adsorbierte wăsserige Lösung, und dieser UeberschuB ist in sämtlichen Experimenten annähernd konstant innerhalb weiter Grenzen der Konzentration von Säuren und Säure-Salzkombinationen, nie aber ist er größer als 1,25 Milligrammole Säure pro $1 \mathrm{~g}$ trockener Gelatine. Bei starken Säuren, die Methylorange schon in ganz geringen Konzentrationen umfärben, wird die lonisation eines Teiles der von der Gelatihe adsorbierten Säure -- dieser wechselt mit den Versuchsbedingungen, aber immer innerhalb der angegebenen Grenzen - so betrăchtlich herabgesetzt gegenüber der freien Säure, daB er die Färbung des Methylorange nicht mehr beeinflussen kann; durch Phenolphthalein kann man ihn noch nachweisen.

In neutraler Lösung steigern Neutralsalze die Quellung der Gelatine, und die adsorbierte Salzmenge ist dann gröBer, als die normalerweise in der adsorbierten Lösung vorhandene; in saurer Lösung aber, und wenn auch nur eine Spur Salzsäure gegenwärtig ist, bewirken die Neutralsalze eine beträchtliche Entquellung der Gelatine, und die Adsorption des Salzes an die Gelatine wird negativ.

\section{Theoretischer Teil.}

Was nun zur Erklärung der vorausgehenden Befunde gesagt werden kann, muB wohl blob als Arbeitshypothese betrachtet werden. da ja die Untersuchungen noch keinesfalls abgeschlossen und auch die Arbeitsmethoden ziemlich roh sind. $\mathrm{Zu}$ allererst wird es wohl nötig sein, sich irgendeinen bestimmten Begriff von der Struktur ciner Gallerte zu machen. Wir schlieben uns dabei der Ansicht jener an, die ein Netzwerk von Gelatinemolekúlen annehmen, die miteinander zusammenhängen, jedoch $Z$ wischenräume von molekularer Dimension zwischeneinander freilassen, die mit Wasser oder wăsserigen L.ösungen ausgefüllt sind; da sich diese im Bereiche molekularer Anziehungskrafte befinden, sind sie als halbfeste Lösungen in der Ge. latine aufzufassen und haben mit dieser einen gemeinsamen Binnendruck. Das Gelatinemolekül, das ja aus einer komplizierten Kette von Amidosăuren besteht, ist besonders dazu geeignet, solche Strukluren zu bilden. Der Bereich der molekularen Anziehungskräfte isı keineswegs großBer als $10 \mu \mu$, und sehr oft sogar wesentlich geringer ${ }^{1}$ ); aber da ungeführ eine zweiprozentige Gelatinelösung nötig ist, um eine zu-

1) Vgl. H. Freundlich, Kapillarchemie (Leipzig 1909), 27 i. 
sammenhängende Gallerte z"l bilden, so müBte eine Gelatine vom Volumen $(10 \mu \mu)^{9}$ dreizehn Millionen mal schwerer sein, als ein Wasserstoffmolekül; das sind Moleküle, groß genug für ein Netz von molekularen Dimensionen. Die Feststellung, dab die Konzentration der Gelatine im Augenblick des Erstarrens auf die GröBe der darauffolgenden Quellung von Einflut ist, gibt der Anschauung von der Entstehung eines molekularen Netzwerkes eine bedeutende Stūtze.

Diese unsere Auffassung unterscheidet sich wesentlich von denen J. M. van Bemmelen's und O. Butschli's, obwohl sie scheinbar viele Aehnlichkeit mit ihnen aufweist; denn diese Forscher nehmen eine zeltułare Struktur von mikroskopischen Dimensionen an, die also weit außer dem Bereich molekularer Kräfte liegen muB. DaB eine solche zellige oder Pseudo-Gelatine existiert und dargestelit werden kann, ist nicht $z$ leugnen, aber meiner Meinung nach ist es vollkommen unbewiesen, dab eine derartige Struktur von Natur aus in wässerigen Gelatinegallerten besteht, denn alle mikroskopischen Beobachtungen wurden an geharteten und durch Wasserentziehung gegeschrumpften Gelatinen gemacht, wăhrend ungehărtete Gelatinen, in denen die Elemente ja viel größer sein müBten, eine Struktur überhaupt nicht nachweisen ließen. Eine verdūnnte Gelatinelösung, die genügend erhitzt worden ist, um sich ganz zu lösen, zeigt nur vereinzelte und wahrscheinlich zufällige Submikronen und nur eine schwache Tyndallwirkung unter dem Ultramikroskop, so daB sich meistens eine echte molekulare Losung annehmen läBt, bei der die Gelatine gleichmäBig in der Flūssigkeit verteilt ist. Beim Abkühlen findet keine Ausflockung oder sichtbare Kontraktion der Gelatine statt, sondern das Ganze wird zu einer transparenten und offenbar homogenen Gallerte, und man kann sich schwer vorstellen, wie unter diesen Umständen eine zellige Struktur entstanden sein konnte. In einigen anderen Fällen, wie z. B. bei der. Hitzegerinnung von Albuminlösungen, wo das Gel offenkundig weniger hydrophil ist als das Sol, muB sich das Netzwerk, selbst wenn es zu Anfang gleichformig und molekular gewesen ist, notwendig zusammenziehen und es muB sich eine Netzstruktur bilden; trennt sich jedoch ein Flüssigkeitsgemisch in zwei teilweise unmischbare Flüssigkeiten, dann entsteht eine Emulsion, die eine Pseudo-Emulsion sein kann; aber keine dieser Bedingungen scheint bei der Gelatine zuzutreffen, und die Annahme einer zelligen Struktur vermag keineswegs die beobachteten Tatsachen zu erklären, sondern im Gegenteil, sie erschwert ihr Verständnis bedeutend. 
Akzeptieren wir die Erklărung, daB eine Gelatine die Lossung einer Flüssigkeit in einem elastischen festen Körper ist, so spitzt sich natürlich das Problem auf die Frage des osmotischen Druckes zu, oder genauer gesagt, auf die Frage einer Verteilung zwischen zwei nicht mischbaren Lösungsmitteln. In diesem letzten Falle nimmt die gemeinsame Oberflăche die Stelle einer semipermeablen Membran ein, und da die Lősungsmittel nicht mehr identisch sind, spielt auch der Teilungskoeffizient und manchmal auch eine verschiedene molekulare Komplexităt eine Rolle. Noch ein wichtiger Unterschied, der leicht übersehen werden könnte, besteht jedoch zwischen beiden Fallen: Bei der gewöhnlichen Osmose dienen die molekularen Anziehungskrăfte des gemeinsamen Lösungsmittels nur dazu, die des gelösten Stoffes zu überwăltigen und in ein Gleichgewicht $z u$ bringen, so da 8 in der Losung schlieblich ein gleicher und gemeinsamer innerer Druck herrscht, dessen kinetische Energie sich gegen eine semipermeable Membran richtet; d. h. in den Ausdrücken der Gasgleichung, dab V konstant bleibt und $P$ variiert. Bei dem Gleichgewichte unmischbarer Flüssigkeiten hingegen ist die kinetische Energie konstant, und es ist die wechselnde innere Anziehung der beiden Lỏsungsmittel für ihre Moleküle, die den Teilungskoeffizienten bestimmt, und da die Diffusionsoberflache frei beweglich ist, so ist hier $P$ konstant, wăhrend $V$ variiert. Diese letztere Feststellung wird bei den Gallerten noch etwas modifiziert durch die Kohäsionskrăte der festen Bestandteile, die einer Volumsănderung entgegenarbeiten. Es labt sich jedoch zeigen, dal diese Kräfte in der Nahe der Region der stärksten Quellung besonders klein sind. P. von Schroeder') zeigte, daB eine Gallerte, die in Wasser gequollen war, in einer Atmosphäre von gesattigtem Wasserdampf einen großen Teil ihres Gewichtes und Volumens verliert (bis 73 Proz.); daB dies, wenn die Gallerte mit einer n. 10 $0^{-5}$ Alkalisulfatlð̋sung getrankt worden ist, sich jedoch vollstăndig vermeiden, und mit einer $10^{-6} n$ Losung erheblich vermindern lieB. Ich ${ }^{2}$ ) habe ferner gezeigt, da $B$ die Energie, die nōtig ist, um $1 \mathrm{~g}$. Wasser gegen den osmotischen Druck einer n. 10-5 Sulfatlösung zu bewegen (350 Erg), sich ganz gut mit der vergleichen läBt, die bei der Bildung einer Kugel von $1 \mathrm{~g}$ Oewicht gegen die Oberflăchenspannung zur Geltung kommt; ohne Zweifel kabt sich auch die Schrumpfung auf diese Weise erklăren. Daraus folgt, daB in den meisten Pällen, wo eine starke

1) Coll. 1803, 204.

2) Brit. Assoc. Rep. 1908, 216. 
Quellung auftritt, die Kohäsion im Vergleich zu den osmotischen Kräften verschwindend gering ist. Andererseits sind auch in den ersten Stadien der Wasseradsorption durch trockene organische Kolloide, einschlieblich der Gelatine, die wirksamen Kräfte sehr groB, wie aus der deutlichen Wărmeentwicklung, aus der Volumskontraktion und aus den sehr beträchtlichen Drucken hervorgeht, die entstehen, wenn der Quellung mechanische Hindernisse entgegentreten.

Nimmt man an, daB die Quellung von Gelatine in reinem Wasser auf Grund osmotischer Wirkungen geschehe, so gilt dies noch sicherer für die dehydrierende Wirkung von Alkohol auf die gequollene Gelatine, da diese für Wasser zwar leicht durchlăssig, für Alkohol dagegen praktisch undurchlasssig ist, so dab die Gelatinemasse wie eine einfache osmotische Zelle wirkt. Die Quellungskurven in Mischungen von Wasser und Alkohol (Tab. 2, Fig. 1) stellen einen einfachen Typus dar; in ihrem mittleren Teile entsprechen sie nahezu einer gleichseitigen Hyperbel, an den beiden Enden weichen sie davon ab, wahrscheinlich infolge der Starrheit der Galierte; es ist kein Grund vorhanden, an der vollstandigen Reversibilitat dieses Vorganges zu zweifeln. Alkohol ruft in einem Osmometer mit einer Gelatinemembran einen beträchtlichen osmotischen Druck hervor, und es wurde auch nachgewiesen, dab Alkohol, der in Gelatine eingeschlossen ist, in ihr eine zellige Struktur erzeugen mub, wobei die Gallerte als semipermeable Membran fungiert. Es ist mogglich, dab ausführlichere Untersuchungen über die dehydrierende Wirkung von Alkohol auf Gallerten einen genaueren Begriff von deren Kohăsions- und osmotischen Kraften geben könnte, da es sich ja hier um rein physikalische Wirkungen handelt.

Viel komplizierter wird es für die Săurequellung und für das spezifische Maximum der Săurequellungskurve, ferner für die Dehydratation der sauren Gelatine durch Neutralsalze eine Erklärung zu geben; wir müssen hier sowohl chemische, als auch rein osmotische Prinzipien heranziehen. Im folgenden wird eine sehr ausführliche Darstellung der Wirkung von Salzsäure und Kochsalz gegeben werden, da diese beiden am gründlichsten untersucht worden sind, und wohl kaum angenommeu werden kann, dab diese Resultate von denen für andere Sauren und ihre korrespondierenden Salze im Prinzip abweichen.

Die Gelatinegallerte ist bekanntlich für Săuren, Salze und für deren. Ionen sehr durchlässig, so daB es nicht leicht einzusehen ist, wieso einer dieser Stoffe einin direkten osmotischen Druck auf die Gelatine auszuüben vermag. Trotzdem ist aber gezeigt worden, daß sogar sehr verdünnte Salzsäure von der Gallerte heftig adsorbiert wird, so 
dą diese dıe Săure immer in einem betrăchtlichen Ueberschuß enthält im Vergleich zum gleichen Volumen umgebender Lösung, mit der sie sich im Gleichgewicht befindet. Dieser UeberschuB nimmt anfangs schnell $z u$, erreicht aber bald einen Punkt, wo er konstant wird (siehe die Kurve der "fixierten Säure“ in Fig. 2); dieser Verlauf legt nun die Idee sehr nahe, daß es sich um einen hydrolysierenden chemischen Komplex mit den Eigenschaften eines Salzes handelt. wobei die amphotere Gelatine als Base wirkt. Diese Annahme wird durch die Tatsache gestützt, daß eine annähernd entsprechende Menge Säuregelatine gegenüber Methylorange neutral reagiert, obwohl sie bei genügendem Ueberschuß von Wasser noch weiter zu hydrolysieren vermag und obwohl diese durch Titration mit Phenolphthalein als Indikator gegen kaustisches Alkali bestimmt werden kann. Existiert ein solches Gelatinechlorid, so ist es sehr wahrscheinlich, dab es für Salzsăure und andere Chloride viel weniger durchlăssig sein wird, als die neutrale Gelatine, oder aber, wenn wir die Gallerte als Losung betrachten, daB die Löslichkeit von Salzen mit dem gleichen Anion in saurer Gelatine viel geringer sein wird als in neutraler, während ihre Affinităt für Wasser - vielleicht wegen der stårkeren lonisation -- gröBer sein wird. Die Gallerte muß sich natürlich in jeder Beziehung mit der umgebenden Lösung im Gleichgewicht befinden, vor allem in bezug auf die Hydrolyse, wobei sie dem gewöhnlichen Massenwirkungsgesetze folgen wird. In bezug auf das Volumen haben wir es bier mit denselben Erscheinungen zu tun, wie wenn Lösungen einer Săure und deren Salze durch eine bewegliche Membran voneinander getrennt sind, die wohl für die Săure und das Wasser, nicht aber für das Salz durchlassig ist und die in unserem Falle durch die keine Diffusion gestattende Kolloidgallerte dargestellt wird. Die beiden Lösungen müssen sich in vollständigem Gleichgewicht befinden, und da das Salz nicht diffundieren kann, müssen Wasser und Säure so lange die Membran passieren, bis das Gleichgewicht erreicht ist. Dann müBte sich aber vor allem das gemeinsame Anion in beiden Lösungen in der gleichen Konzentration befinden, und wegen der Salzanionen muB die freie Săure in der salzhaltigen Lösung notwendig weniger konzentriert sein, als in der salzfreien. Es müssen aber auch die Säureanionen in der Salzlosung sich mit den Anionen des Salzes selbst im Gleichgewicht befinden, und das kann nur dann geschehen, wenn Wasser und Săure so lange adsorbiert oder abgestoßen werden, bis der. lonisationsdruck des Salzes dem der Săure, die in der Salzlősung enthalten ist, gleichkommt. Auf diese Weise sind die nötigen 
Daten gegeben, um das Volumen der Salzgallerte genau zu bestimmen: es ist von der Konzentration der Săureionen und von der lonisation und der Quantităt des Salzes abhängig. Dịe Menge des nicht hydrolytisch gespaltenen, aber ionisierbaren Gelatinesalzes hingegen hängt für eine bestimmte Menge der Base von der $\mathrm{H}$-lonenkonzentration der Săure und von der Hydrolysenkonstante des Salzes ab; derart konnen Săuren mit verschiedenen Konstanten sehr mannigfache Quellungseffekte hervorbringen.

Eine Konsequenz der obigen Auseinandersetzungen muB noch berücksichtigt werden. Es ist nämlich leicht einzusehen, daß die -fixierte Säure“, die unter der Annahme berechnet wurde, dab die adsorbierte Lösung die gleiche Säurekonzentration habe, wie die umgebende Säure, nicht dem gesamten unhydrolysierten Gelatinesalz. entspricht, sondern dab sie um so viel geringer ist, als die Säuremenge betrug, die durch das Salz "aus ihrer Losung verdrängt wurde. Ob nun die ganze Säure durch das ionisierte; oder auch ein Teil durch das nicht ionisierte Salz verdrängt wird, das zu seiner Auflösung Wasser braucht, ist nicht leicht zu entscheiden, aber wohl auch für die allgemeine Gleichgewichtstheorie nicht von Bedeutung.

- Es ist natürlich unmöglich, das Problem schon jetzt streng mathematisch zu behandeln, wo ja noch so viele Faktoren unbekannt sind, vor allem die GesetzmäBigkeiten der Kohăsionselastizität der Gallerte; aber es ist doch wohl interessant, zu-untersuchen, inwieweit sich die Versuchsergebnisse mit den theorethischen Annahmen in Uebereinstimmung befinden.

Wir haben angenommen, daB die Verbindung von Gelatine mit Salzsăure den Charakter eines Salzes einer schwachen Base mit einer starken Saure hat, deren Hydrolyse sich nach Wilh. Ostwald durch die Gleichung $\frac{h B}{b}=K_{g}=k$ ausdrücken läBt, wobei $h$ die $H$ lonenkonzentration, $\mathrm{b}$ die Konzentration der kolloiden Gelatineionen und $B$ die durch Hydrolyse entstandene, aber nicht elektrolytisch zerfallene Gelatinebase bedeutet. $K_{\mathbb{v}}$ ist die Dissoziationskonstante des Wassers, $K_{g}$ die der Gelatine als Base. Man kann nun ohne betrăchtliche Fehler annehmen, dab die Salzsäure in den Verdünnungen, um die es sich hier handelt, vollkommen ionisiert ist und dab daher die Säurekonzentration $x$ in der Lösung den $\mathrm{H}$-lonenkonzentrationen $h$ proportional ist. $b$ ist die Konzentration des Kations des ionisierten, aber nicht hydrolysierten Salzes, und da die Menge der Gelatine in dem Experiment konstant ist, so ist B offen- 
bar gleich $1-b$. Die obige Gleichung nimmt daher folgende Form an : $\frac{(1-b) x}{b}=k$; und wenn wir diese Gleichung nun nach $b$ auflosen, so erhalten wir $b=\frac{x}{x+k}$ als das MaB des nicht hydrolysierten Anteils des Salzes ${ }^{1}$. Den gleichen Ausdruck kann man aber auch aus dem gewöhnlichen Verdünnungsgesetz $\frac{a^{2}}{(1-a) v}=k$ ableiten, das sich, wenn man die Dissoziationskonstanten durch die hydrolytischen ersetzt, auf mäBige Hydrolyse gerade anwenden läbt. In dieser Formel entspricht a dem bydrolysierten Anteil, den wir oben 1-b genannt haben. 1 - $a$ ist dann das nicht hydrolysierte Salz b, wăhrend die Konzentration der hydrolysierten Säure gleich $\mathbf{x}$ ist, also gleich der der ăuberen Lossung. Setzen wir diese Werte ein und lősen wir wieder nach $b$ auf, dann erhalten wir wieder die Formel $b=\frac{x}{x+k}$. Man kann sehen. $\mathrm{da} B$ in dieser Formel $\mathrm{v}$ nicht vorkommt, da die Konzentration durch $x$ ausgedrückt wird und daher kann man schließen, daß die Hydrolyse durch das Volumen der Gallerte nicht beeinfluBt wird. Der Wert für $b$ steigt anfangs rapid an und strebt dann einem einheitlichen Werte zu; das Steigen geschieht um so schneller und der spătere Teil vorläuft um so mehr horizontal, je geringer der Wert von $k$ ist. Man darf auch nicht vergessen, daB die Ostwald'sche Gleichung nur eine approximative ist und besonders bei Annăherung an eine vollständige Hydrolyse ungenau wird.

Der einheitliche Wert, dem unsere Formel zustrebt, ist der eines Moleküls oder Aequivalents eines Salzes. In den vorliegenden Experimenten wurde $1 \mathrm{~g}$ Gelatine verwendet, von der man ja weder das Molekulargewicht noch die Valenz kennt, und es ist ganz leicht möglich, daß die Wertigkeit sich ändert, sobald die Säure stărker konzentriert wird und dann. wahrscheinlich neue Aminogruppen angreift ${ }^{2}$ ). Es ist unmoglich, aus den Versuchen mit Salzsäure direkt die Aequi-

1) Der hydrolysierte Antell ist offenbar $\frac{\mathbf{k}}{\mathbf{x}+\mathbf{k}}$.

9) Es muB noch bemerkt werden, daB. die Zahien in Tab. 3 und 4 ein plotzliches und erhebliches Ansteigen sowohl der gesamten Saure als auch der ,fixierten Saure* (wie sich aus dem Volumen der adsorbierten Losung berechnen lieB) bei Konzentrationen von mehr als 0,2 $\mathrm{n}$ zeigt; obwohl man dies ja auch auf einen Versuchsfehler zurlickfahren kónnte, ist es doch wahrscheinilicher, daB dadurch angezeigt wird, daB in diesen Konzentrationen weitere Aminogruppen der Gelatine angegriffen werden, entsprechend ihrer erhohten Loslichkeit in der Săure. 
valentgewichte $z u$ berechnen, da bei der Quellung ein großer Teil der freien Såure adsorbiert wird und diese, wie oben gezeigt warde, nicht die gleiche Konzentration hat, wie die AuBenflüssigkeit. Bei sehr verdünnten Săuren ist der Fehler nicht bedeutend, da ja die gesamte Săuremenge in der adsorbierten Lősung klein ist, im. Vergleich zu der, die durch die Gelatine fixiert wurde, aber in diesem Falle ist die Hydrolyse auch sehr grob. Es wurde jedoch gezeigt, daB sogar bei Verwendung von Săuren in stärksten Konzentrationen die Quellung betrăchtlich vermindert werden kann, wenn man einen ausreichenden UeberschuB eines Salzes mit gemeinsamem Anion zufügt (z. B. Natriumchlorid), und da das gleiche Anion die Hydrolyse nicht vermehrt, sondern herabsetzt, indem ja die lonisation des Salzes vermindert wird, so kőnnen wir wohl annehmen, daß die Hydrolyse in diesem Pall nahezu gleich Null ist. Wir finden, daB unter diesen Umstånden die Gesamtmenge der durch $1 \mathrm{~g}$ Gelatine adsorbierten Saure ungefăhr $1,3 \mathrm{mg}$ Mole betrăgt, während sie in der adsorbierten Lossung 0,05 mg Mole meistens nicht übersteigt. Wir können also ungefăhr annehmen, dab unter diesen Bedingungen $1000 \mathrm{mg}$ Gelatine sich mit 1,28 mg Molen Săure verbinden, oder, mit anderen Worten, daB das Aequivalent- (wenn auch nicht gerade das wirkliche Molekular)gewicht der Gelatine, wenn wir sie als einwertig auffassen, ungefăhr 780 betrăgt.

Wir können uns jetzt der weiteren Frage zuwenden, inwieweit das Quellungsvolumen der Gelatine mit der Saureadsorption zusammenhängt. Die experimentelle Kurve ist sehr aigenartig, sie weist ein deutliches Maximura auf, aus-dem sich ersehen laßBt, daB die Quellung bei zunehmender Konzentration zuerst stetig ansteigt, hernach aber - und zwar etwas langsamer - wieder abfallt. Nach der Theorie, die wir hier auseinandergesetzt haben, ist die Quellung auf die größere Loslichkeit oder Wasseraffinităt des ionisierenden Gelatinehlorids im Vergleiche mit der nicht ionisierenden neutralen Gelatine zurückzuführen, und sie muß daher zugleich mit der vermehrten Entstehung des Chlorids anwachsen. Andererseits wird sie auch durch die Anionen der Săurelösung gehemmt, die nicht nur durch Verdrungung von Wasser die Konzentration der Gelatine vermehren, his ihr gesamter lonendruck dem der Saure gleichkommt, sondern auch die Verdrăngung frefer Săure aus der Gelatine gegen denselben Druck $\mathrm{x}$ bewirken, so daB der gesamte Anionendruck der Gallertmasse gleich $\mathrm{x}$ bleibt. Daher verăndert sich die Kraft, die nötig ist. um die Gallerte zu komprimieren, nicht mit $x$, sondern mit $x^{2}$, und 
die Quellung müBte daher bei einer gegebenen Vermehrung von $x$ abnehmen wie $\boldsymbol{V}_{\bar{x}}$. Es wäre daher zu erwarten, daB sich die Quellung etwa durch eine Formel wie $\beta \ldots x+k / V_{x}$ oder $\beta \frac{\sqrt{x}}{x+k}$ ausdrücken läßt, wobei $x$ und $k$ dasselbe wie oben bedeuten, und wo $\beta$ eine empirische Konstante darstellt, die die Beziehung des Volumens der Gelatine zu ihrem Druck zum Ausdrucke bringt. Eine solche Formel wird offenbar bei irgendeinem kleinen Wert von $x$ ein deutliches Maximum erreichen, und durch Differenzieren kann man zeigen, daß dieses Maximum dann eintritt, wenh $x$ gleich $k$ ist. Wir haben so ein Mittel, zu einem Werte für $k$ zu gelangen, da ja das experimentelle Maximum deutlich bei $x=0,005 \mathrm{n}$ liegt, und so ist die Quellungskurve in Fig. 2 als $7,8 \frac{\sqrt{x}}{x+k}$ berechnet. Es ist möglich, daß der wirkliche Wert für $k$ etwas kleiner ist als 0,005 $n$ und daB das höhere Maximum, das so hervorgerufen werden maßte, durch die Kohāsion der Gallerte verhindert wird; und da die Kurve viel plotzlicher ansteigt, als sie abfallt, so wtirde die Wirkung dieser Abrundung des Kurvengipfels darin bestehen, daß das deutliche Maximum auf eine höhere Konzentration verschoben wird; wenn aber die Hypothese korrekt ist, so kann es auf keinen Fall viel niedriger als bei $0,005 \mathrm{n}$ liegen, jedenfalls wird die betreffende Konzentration von dieser Größenordnung sein. $\mathrm{Da}$ ja $k=\frac{\mathrm{K}_{\mathbf{V}}}{\mathrm{K}_{\mathrm{E}}}$ ist und wir $\mathrm{K}_{\mathbf{W}}$ kennen, kơnnen wir für die Dissoziationskonstante der neutralen Gelatine einen annähernden Wert berechnen, er betrüge $\frac{0,6 \times 10^{-14}}{0,5 \times 10^{-2}}$, wăre also von der GröBenordnung $1 \times 10^{-12}$. Es wăre interessant, dies durch direkte Messungen zu bestätigen.

Von den direkten Versuchsergebnissen bleibt jetzt nur noch der Wert für die Gesamtmenge der adsorbierten Săure zu berechnen. Diese erhălt man (auf die Art wie die "fixierte Săure" bestimmt wurde) offenbar dann, wenn man die Summe aus dieser und der Menge der in der Gallerte adsorbierten Flüssigkeit mit der Konzentration der auferen Losung multipliziert. Da die erstere ungefahr 0,8 Mole beträgt - im Momente der maximalen Quellung -- und die letztere $x \frac{7,8 y x}{x+k}$, so wurde die Kurve in Fig. 2 als Summe dieser beiden entworfen; sie stimmt mit den experimentellen Werten recht gui überein, gibt jedoch keine ErkJärung för die Menge und Konstanz der fixierten Săure“. Die Prage wird im năchsten Abschnitt 
besprochen werden. Immerhin ist es interessant, darauf hinzuweisen, dab die Kurve der adsorbierten Säure ebenso durch die Gleichung $\eta==87 . \times$ 0,41 ausgedrückt wird; die logarithmische Darstellung dieser Kurve ist nur leicht konvex und zeigt so, daB Kurven, deren Anstieg die Funktion rein chemischer Vorgänge ist, ganz nach dem Typus von Adsorptionskurven verlaufen.

Es ist angenommen worden, daB der Widerstand, den die Gallerten einer Kompression durch die Säure der äußeren Lösung entgegensetzen, das Produkt aus dem direkten lonendruck des Gelatinesalzes und dem des Anions der Säure ist, die mit dem Wasser zugleich verdrängt wird, um das Gleichgewicht zu erhalten, die jedoch ihre korrespondierenden H-Ionen mit sich führen muB. Der Quellungsdruck im Innern der Gelatine ist somit aus drei Partialdrucken zusammengesetzt, von denen nur einer von dem Anion selbst ausgeübt wird; die verdrängte Säure bildet daher nur ein Drittel des gesamten ionisierten Gelatinesalzes. Da zwischen $x=0,5$ und $x=0,3$ die Menge und lonisation des Gelatinechlorids, soweit man es beobachten kann, vollständig und konstant bleibt, und da jeder Wechsel, der in einem Teile stattfindet, durch einen entsprechenden Wechsel im andern teilweise kompensiert wird, so wird die "fixierte" und die verdrängte Săure ebenfalls konstant bleiben: die Konzentration der einen liegt bei 0,8 , die der andern bei 0,4 Molen. Wir wissen his jetzt noch nichts Bestimmtes über die lonisation des Gelatinesalzes, deren Zurückdrăngung in den hier angeführten Grenzen wohl vernachlässigt werden kann, bei Gegenwart von $\mathrm{NaCl}$ oder einem andern Salze mit gemeinsamem Anion jedoch recht bedeutend werden muB. In diesem Falle wird die Menge der "fixierten Saure" als unionisiertes Gelatinesalz anwachsen und die der verdrängten Säure wird geringer werden, so dab die Kurve der "fixierten Säure" sich immer mehr der der Konzentration des unionisierten Gelatinesalzes nähert, was ja unter diẹsen Umständen auch experimentell nachgewiesen wurde.

Ein Punkt, dessen Erklärung viel mehr Schwierigkeiten macht, ist der nahezu vertikale Anstieg der Kurve der "fixierten Säure" in der Nähe des Anfangs und das leichte Maximum derselben entsprechend dem Maximum der Quellungskurve. Es ist klar, dak die wirklich mit der Gelătine in Verbindung getretene Säuremenge nicht größer sein kann, als es nach der Hydrolysengleichung möglich ist, und daß für den vermehrten Anstieg der Hydrolyse keine Konstanten oder Modifikationen gefunden werden können, die die daraus abgeleiteten Quellungskurven nicht vollkommen,auber Einklang mit dem Experiment 
brächten. Es ist eine vielleicht etwas forcierte, aber wohl nicht vollkommen unwahrscheinliche Erklärung, daB die unkombinierte Gelatine zuerst Säure adsorbiert, ohne sie jedoch innerlich zu binden, und dab diese Adsorption durch das grobe Volumen der Gallerte in dem Stadium der maximalen Quellung begünstigt wird, wo ja auch die Kurye der „fixierten Säure" ein niedriges Maximum aufweist. Diese Adsorption würde natürlich dahin zielen, die Fixation von Săuren zu vermehren und einheitlicher zu gestalten und würde zugleich mit der freien Base verschwinden, da es unwahrscheinlich ist, dab das Chlorid noch. Salzsäure adsorbiert. Es wären wohl noch einige andere Erklărungen diskutabel; aber es wird besser sein, die Ergebnisse weiterer Experimente abzuwarten.

Man mub, wie ich glaube, zugeben, daß das komplizierte System, das aus der Theorie der wirklichen chemischen Verbindungen der Gelatine abgeleitet wurde, mit den experimentellen Befunden eine weitgehende Uebereinstimmung zeigt, die mehr als zufällig ist und die in gewisser Hinsicht wirkliche Tatsachen darstellt, mögen diese nun im strengsten Sinne chemische Vorgănge sein oder nicht. Es läBt sich nicht ohne weiteres leugnen, daB eine ebenso plausible Hypothese sich auch auf die physikalische Adsorption stützen lieBe; aber um befriedigend zu sein, müBte sie einen zumindest ebenso vollständigen Zusammenhang mit den Versuchsbefunden aufweisen wie die hier ausgeführten Gedanken. Wir müssen zugeben, daß noch viele unklare Abweichungen erklärt werden müssen, indem weitere Beispiele beigebracht werden, und indem an Stelle unserer empirischen Konstanten solche gesetzt werden, die mit unseren sonstigen Kenntnissen in Zusammenhang gebracht werden können; immerhin aber ist durch die vorliegende Untersuchung wenigstens eine Arbeitshypothese gegeben. Die wesentlichste Abweichung der Tatsachen von unserer Theorie besteht im rapiden Anstieg der Menge der "fixierten Saure* zu einem kleinen Maximum, das höher liegt, als es nach der Konzentration des hydrolysierten Salzes anzunehmen wăre; wenn man die wahrscheinllchen Pehler des Verdünnungsgesetzes in einem so extremen Falle vernachlassigt, so ist es naheliegend, daB das obige Phänomen durch physikalische Adsorption erklărt werden kann, die der der chemischen Bindung vorausgeht.

Man sieht, daB uns die Theorie eine vollstăndige qualitative Erklärung für die dehydrierende Wirkung von Kochsalzlosungen und anderen Chloriden auf Gelatine (und ebenso auf die Haut und Bindegewebe) liefert, die mit Salzsăure quellen gelassen wurde, obwohl 
ja unsere Kenntnisse über die Gesetzmäbigkeiten in konzentrierten Lossungen nicht dazu ausreichen, dab wir dieselbe Gleichung auch quantitativ verwenden könnten. Durch die Theorie ist sichergestellt, daB die Abnahme der Quellung durch den Druck der Chlorionen hervorgerufen wird, wobei es gleichgültig ist, ob diese durch Säuren oder durch ein anderes Chlorid geliefert werden. DaB Salze nicht nur einen Kompressionsdruck auf die Chloridgallerte ausüben, sondern auch durch diese aus der adsorbierten Lösung ausgetrieben werden, beweisen He Experimente in der letzten Kolonne in Tab. 7 und 8, aus denen hervorgeht, dab die Fixation des Salzes negativ ist, obwohl ja bei Anwesenheit von soviel Salz die Schătzung der Chlorionen nicht so genau vorgenommen werden kann wie die der Saure. DaB Salze auf neutrale Gelatine keine kontrahierende, sondern eher eine quellende Wirkung ausüben, geht aus Tabelle 11 hervor, wo ja auch die Salzadsorption positiv ist. Die Salzwirkung, die darin besteht, die Menge der "fixierten Săure" zu vermehren, ist auch leicht zu erklăren: die Gegenwart von Chlorionen beschrănkt die Hydrolyse, indem sie die lonisation herabdrückt und so die Menge des nicht ionisierten Chlorides vergrößert und die Verdrängung der Säure aus der adsorbierten Lösung dadurch vermindert, daB es selbst ausgetrieben wird. Es entsteht immerhin noch die Frage, ob die Entquellung der Gallerte unter diesen Umständen blob als ein Fall ionischen Gleichgewichtes zu behandeln ist oder nicht eher als ein "Aussalzungsprozeb “, bei dem die Avidităt der verschiedenen Bestandteile für Wasser mehr ins Gewicht fallt, als ihr lonendruck, und bei dem das unionisierte $\mathrm{NaCl}$ ebensogut eine Rolle spielt wie das ionisierte. In unseren Gleichungen haben wir durchweg angenommen, dab die Gesamtmenge des Gelatinechlorids und nicht nur sein ionisierter oder nichtionisierter Anteil bei der Quellung wirksam ist; wenn aber die lonisation der Gelatinesalze mit der der meisten Salze vergleichbar ist, so muB sie offenbar praktisch vollkommen sein, wenigstens bis zu jenen Konzentrationen, wie sie in unseren Săureversuchen verwendet wurden.

Jetzt erübrigt es uns noch, zu ubberlegen, ob die Theorie, die wir aus dem Studium der Wirkung der Salzsãure abgeleitet haben, für alle Arten der Säurequellung von Gelatine oder gelatinösen Geweben anwendbar ist, oder ob sie nur einen Ausnahmefall darstellen, der vielleicht nur für wenige stärkere Säuren gilt, wie ja auch Schwefelsăure und Sulfate eine den entsprechenden Chloriden vollkommen analoge Wirkung aufweisen (siehe Tab. 14). 
Es ist oft festgèstellt worden, dab̆, da alle Säuren von einer genügenden H-Ionenkonzentration den gleichen Quellungseffekt aufweisen, die $\mathrm{H}$-lonen als das wirksame quellende Moment angesehen werden müssen. In dem Lichte unserer Theorie betrachtet, kann dies jedoch nur indirekt der Fall sein, insofern die H-lonenkonzentration das Maf für die Avidität der Gelatine ist und daher auch für deren Fähigkeit, Salze zu bilden; so ist es ganz leicht mơglich, daB, wie dies ja bei Ammoniumsalzen und den Salzen vieler anderer organischer Basen der Fall ist, nicht nur das Anion, sondern auch das. H-Ion in das Salz eintritt. In allen Fällen ist das Anion das komprimierende und dehydrierende Agens, so daB bei Abwesenheit eines neutralen Salzes die Säure selbst ein Maximum und darauffolgend eine Kontraktion hervorruft. Deutlich ist das nur bei den stärkeren Säuren, da eine schwache Säure, obwohl sie ja imstande ist, ein Gelatinesalz hervorzubringen, doch so wenig ionisiert ist, daB ihre Anionen keine genügende Konzentration erreichen, um den Druck des stărker ionisierten Gelatinẹsalzes zu überwinden. So bringt Schwefelsăure ein deutliches Maximum hervor; das der Ameisensăure ist zwar noch erkennbar, aber viel weniger deutlich, bei Essig- und Milchsäure ist überhaupt kein Maximum mehr vorhanden, die Quellung nimmt mit wachsender Konzentration zu, wird gegen den Schluß stärker als selbst bei starken Säuren und geht ohne Unterbrechung bis zur schließlichen Losung der Gelatine weiter. In diesen Fällen ist die Menge der fixierten Săure ein wenig veränderlich, manchmal etwas gröBer, manchmal auch etwas geringer als bei starken Säuren, je nachdem, ob die Ionenkonzentration ausreirht, um die Hydrolyse zu verhindern, oder um neue Aminogruppen anzugreifen.

Man könnte entgegnen, daß Natrium- oder Kaliumchlorid nicht nur auf Salzsäuregelatine heftig dehydrierend wirken, sundern auch auf jede andere in einer Săure gequollene Gelatine, deren $\mathrm{H}$-lonenkonzentration ausreicht, um überhaupt eine Quellung zu verursachen; aber bei geringer Ueberlegung merken wir, daB eine jede solche Kombination eben zu einem vierfachen Gleichgewicht führt, indem jede Säure mit ihren eigenen Neutralsalzen im Gleichgewicht ist. So haben wir bei Essigsäure und $\mathrm{NaCl}$ Gelatineionen, Azetationen und einen enormen Ueberschub an $\mathrm{Na}$ - und $\mathrm{Cl}$-lonen; stellen wir uns nun vor, dab es hier zu Verbindungen kommt, so müssen sich nach dem Massenwirkungsgesetz viel Gelatinechlorid in einem Gleichgewichte befinden mit dem Natriumchlorid und wenig Gelatineazetat im Gleichgewichte mit Natriumazetat. Wir können daraus als Regel ableiten, 
daB, da das Salz sich immer im UeberschuB befindet, die dehydrierende Wirkung eines Salzes einer starken Säure die gleiche ist, ob nun die Säuerung durch eine schwache oder eine starke Säure zustande gekommen ist und vice versa. Mit Essig- oder Ameisensäure kann man daher ebensogut pickeln, wenn man Kochsalz, wie wenn man irgendein Sulfat oder Chlorid verwendet.

Für andere Săuren und ihre Salze ist gezeigt worden, daß die Quellung in allen Pällen durch genügenden Zusatz des neutralen Salzes vermindert wird, aber am deutlichsten ist der Prozeb bei den Salzen der starken Săuren, vielleicht deşhalb, weil bei den schwachen Săuren die lonisation des zugesetzten Salzes nicht ausreicht, um die der Gelatineverbindung selbst herabzusetzen.

Es ist mir in dieser Arbeit keineswegs darum zu tun, die komplizierte Wirkung von Salzen auf die Quellung neutraler Gelatine zu diskutieren, die besonders von Wo. Paudi,F. Hofme is ter, P. von Schroeder u. a. ') untersucht worden sind; jedenfalls aber ist die Erklărung ziemlich überzeugend, daB in vielen Pällen ein Teil des Salzes elektrolytisch oder hydrolytisch gespalten wird, dab sowohl die Săure als auch die Base sich mit der Gelatine verbinden, und daf die derart entstandenen Verbindungen durch die übrig bleibenden Salzlösungen je nach der Konzentration gequollen oder entquollen werden. J. $\mathrm{PaB}\left(\mathrm{er} \mathrm{r}^{2}\right.$ ) hat gezeigt, daB Natriumbisulfat durch Haut gespalten wird, und daß nur das neutrale Salz in der Lösung bleibt; auch für die Chrom-, Eisen- und Aluminiumsalze ist ein ähnlicher Vorgang nachgewiesen, dab nämlich sowohl die Sāure als auch die Base (oder ein basisches Salz) adsorbiert werden. Die Adsorption. der Base kann entweder als Kombination mit offenen $\mathrm{COOH}$-Gruppen aufgefafit werden, oder aber als Bildung eines komplexen Salzes, wie sie durch Ammoniak 'mit anderen Basen häufig gebildet werden; einige von ihnen mogen auch komplexe lonen bilden, deren Zahl durch die des einfachen Salzes in der Lobsung nicht vermindert wird.

Auch die Frage der Alkaliquellung soll hier nicht berührt werden, doch möchte ich darauf hinweisen, daß sie sich, wie aus einigen vorläufigen Experimenten hervorgeht, in gewisser Hinsicht wesentlich von der Săurequellung unterscheidet. So wird z. B. eine Quellung, die

1) Wo. Pauli (Wo. Pascheles), Pfluger's Atch. 71, 336 u. 399 (1898); Wo. Pauli und P. Rona, Hofmeister's Beitr. 2, 25 v. 26 (1902); F. Hofmeister, Arch. f. exper. Path. u. Pharm. 24, 424 (1888); P. von Schroeder Collegium 1902, 306.

2) J. Pa B ler, Wissenschaftliche Beilage des Ledermarkt, Heft 11, 106 (1901). 
durch Natriumhydrat hervorgerufen worden ist, durch Kochsalz keineswegs vermindert, wohl aber durch höhere Konzentrationen des Hydrats; daraus kann man schließen, daß in diesen Pallen die Hydroxylionen und nicht das Kation $\mathrm{Na}$ bei der Quellung und deren Rückbildung wirksam sind. Ich hoffe, die Frage der Alkaliquellung noch weiterhin verfolgen zu können.

Es ist wohl einleuchtend, daB die in dieser Arbeit diskutierten Tatsachen auch für viele physiologische Fragen von Wichtigkeit sind; in dieser Verbindung möchte ich einen rohen Versuch erwăhnen, der in den ersten Stadien der Untersuchung gemacht wurde und der als ein Abbild der Vorgänge bei der Muskelkontraktion hingestellt werden soll. Eine schwache spiralige Platinelektrode wurde in einen Gallertzylinder eingebettet, dieser dann in eine Salzlösung getaucht, in die die zweite Elektrode gebracht wurde. Machte man nun die Gelatineelektrode zur Anode eines elektrischen Stroms, der genügend stark war, um das Salz elektrolytisch zu spalten, dann wies die Gelatine eine wunderbare Kontraktion auf; ihre Lősung jedoch durch den entgegengesetzten Strom war, infolge der Entwicklung von Wasserstoff, der die Gelatine sprengte, minder befriedigend. Ohne Zweifel müBte man aber mit einer geeigneten Depolarisationsvorrichtung bessere Resultate erzielen und es ist ganz leicht moglich, daß schon die Kondensation des Anions durch eine bloBe Oberflächen-Potentialdifferenz ausreicht, um diese Wirkung hervorzubringen.

Wo. Paulil) hat vor kurzem eine Arbeit über die EiweiBkorper publiziert, in der er ahnliche Ansichten aussprach, wie ich hier, nur legte er ein großeres Gewicht auf die Hydratation der kolloiden lonen. Im besonderen betont auch er sehr stark eine mclekulare Struktur der Gelatine im Gegensatz zu der von $O$. Bütschli angenommenen zellularen oder Netzstruktur. Er erwăhnt ferner auch die Tatsache, daB saure Gallerten nicht durch puren Alkohol dehydriert werden können, was ja auch ich in diesen Untersuchungen fand, obwohl ich beobachten konnte, dab man mit angesăuertem Alkohol beträchtliche Entquellungen erzielen kann.

1) Wo. Pauli, Koll.-Zeitschr. 7, 241 (1910). 